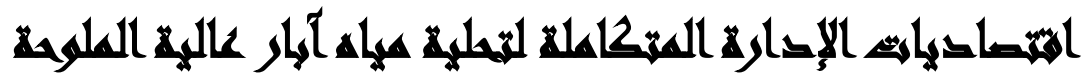

\section{هالأراشيه السمبراوية}

[17]

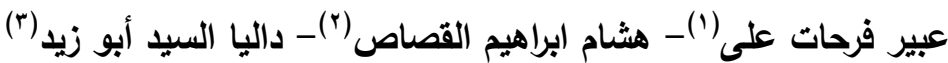

$$
\begin{aligned}
& \text { محمد عمر عبد العزيز محمد الهاصد }
\end{aligned}
$$

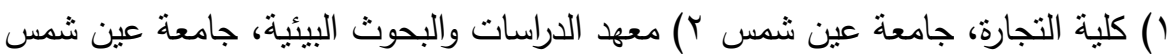

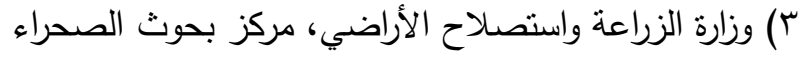

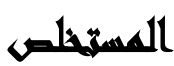

استهذف البحث دراسة اقتصاديات إدارة المحطات المصغرة لتحلية مباه الآبار عالية

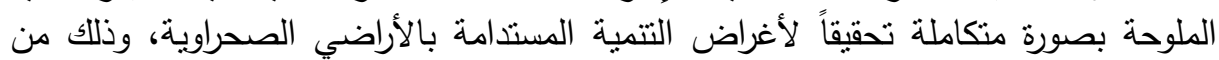
خلال دراسة آليات تطبيق أسلوب الإدارة المتكاملة لمحطات تحلية مياه الآبار عالية الملوحة،

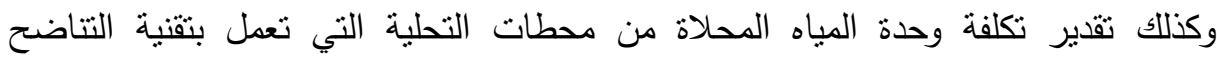

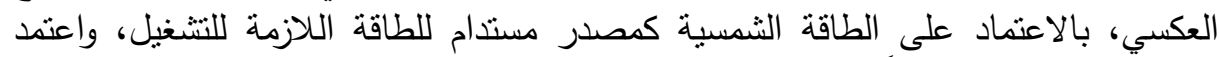

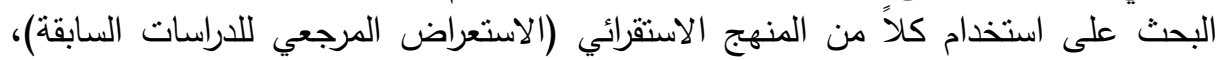

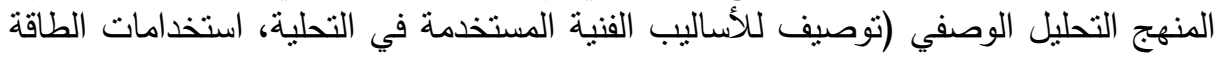

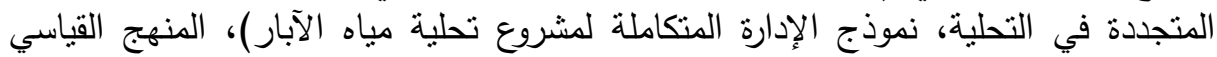

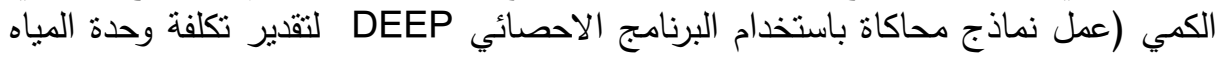

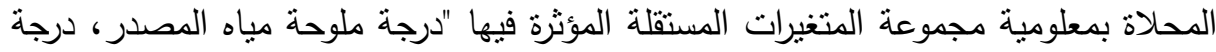

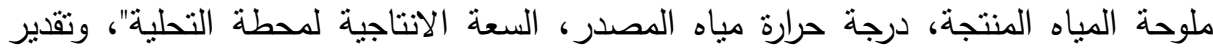

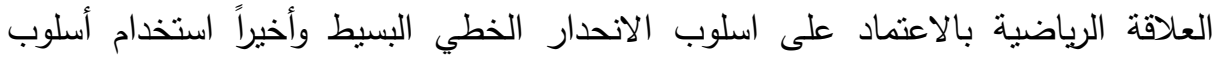

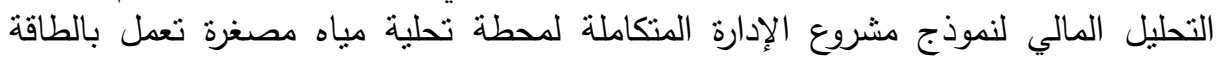

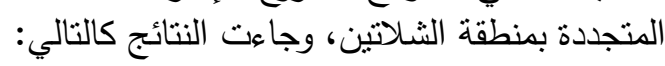

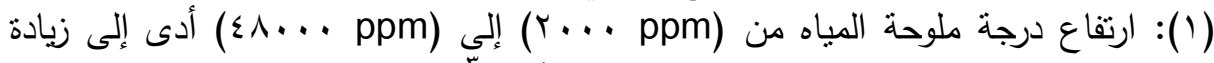

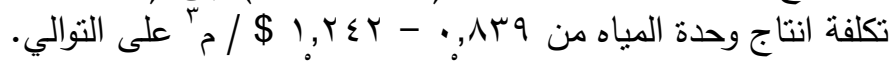

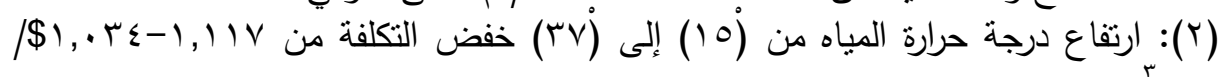

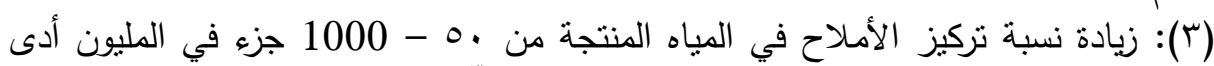

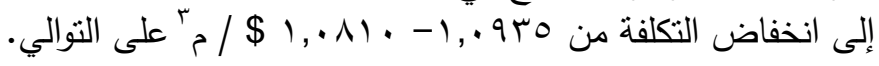

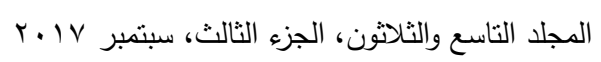




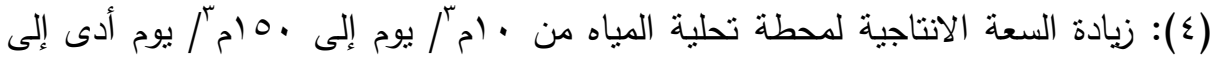

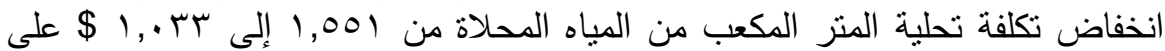

(0): بتقدير الربحية المالية لمشروع الإدارة المتكاملة لمحطة تحلية مصغرة تعمل بالطاقة

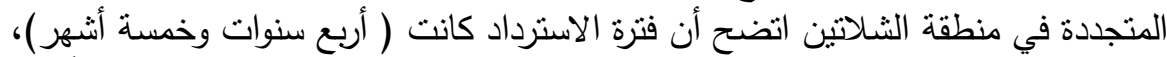

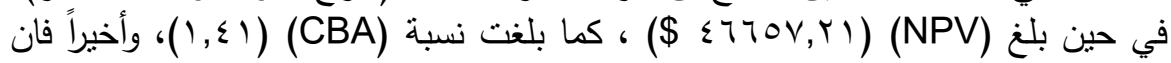

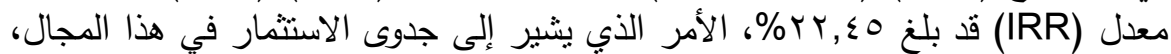

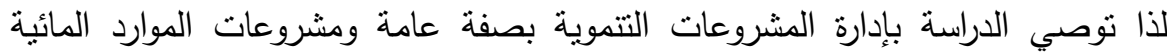

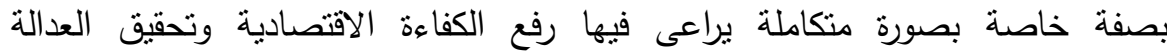

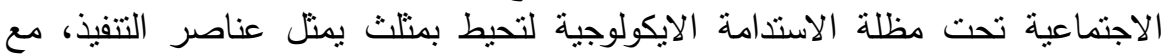

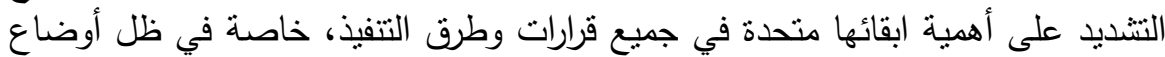
شح المياه بالمناطق الجافة وشبة الجافة. الكلمات المفتاحية: اقتصاديات التحلية-التتاضح العكسية-الثنح المائي - نماذج المحاكاة.

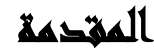

يعد تحدي شح الموارد المائية مشكلة عالمية نواجه الكثير من الدول خصوصاً تلك التي تقع في حزام المناطق الصحراوية الجافة وشديدة الجفاف، لذا فقد أولتها العديد من الدول جانباً كبيراً من عنايتها لتتمية هذه الموارد والمحافظة عليها من عوامل الهدر والتلوث بقدر اهتمامها بتدبير موارد مائية اضافية واتخاذ كافة السبل اللازمة لتعظيم العائد من استخدامات تلك

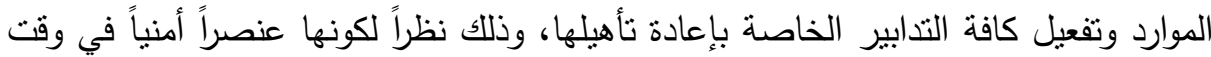
تعتبر فيه مشكلة نوفير المياه واحدة من أكثر المشاكل تعقيداً على المستويات الدولية والإقليمية، وذلك لندرة هذا المورد والذى تزداد حدته بسبب الارتفاع الكبير في الطلب عليه،

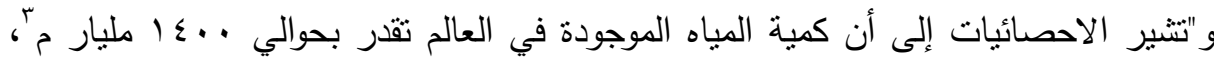
تتكل المياه العذبة منها حوالي ب\% فقط، أما النسبة الغالبة تمثل مياه البحار والمحيطات وتقدر بنحو 9\%\%، مع الأخذ في الاعتبار أن ما يقرب من 79\% من المياه العذبة هي مياه

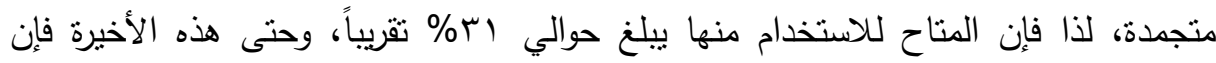

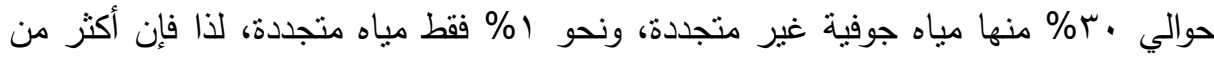


مليار شخص في الدول النامية يعانون من نقص مياه الثرب وخدمات الصرف الصحي"

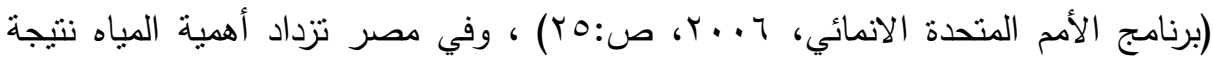
للنبات النسبي في الموارد المائية والذي ينواكب معه الزيادة المضطردة في التعداد السكاني حيث ورد في تقرير مركز المعلومات ودعم القرار أن احتياجات مصر من المياه ستفوق

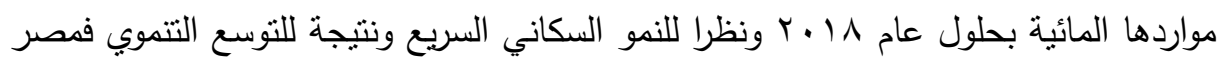

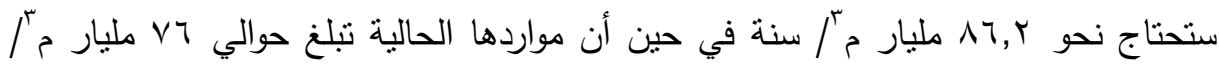

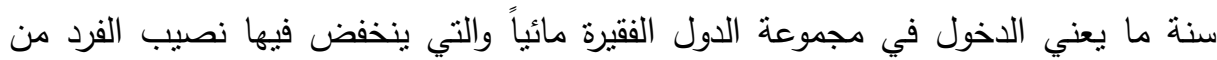

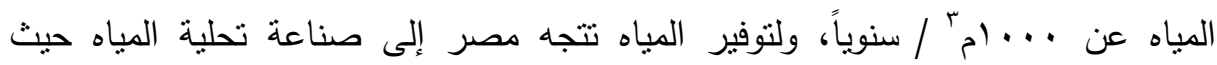
أصبحت تلك الصناعة أحد البدائل التتموية الهامة لمواجهة بعض مظاهر الترفياه الندرة المائية في ظل محدودية الموارد التقليدية في العديد من الأراضي الجديدة.

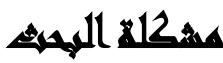

تعد ندرة الموارد المائية أحد أهم المشكلات التي تواجه العديد من الدول التي تقع في حزام المناطق الصحراوية الجافة وشديدة الجفاف، وفي مصر تزداد أهية المياه نتيجة للثبات النسبي في الموارد المائية، حيث يبلغ الإجمالي المنوافر من الموارد المائية التقليدية للمياه

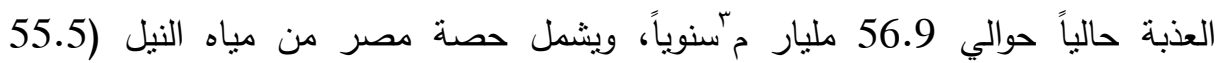

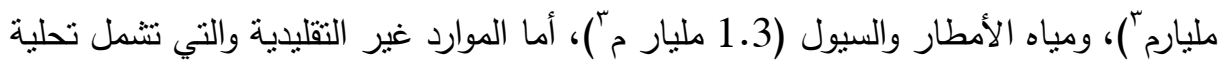

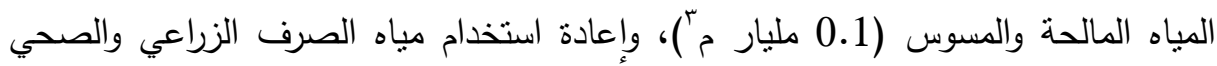

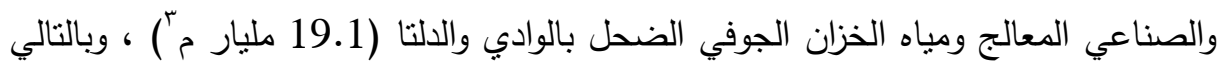
يصل إجمالي المتاح من المياه إلى 76.00 مليار م سنوياً (تقرير الجهاز المركزي للتعبئة

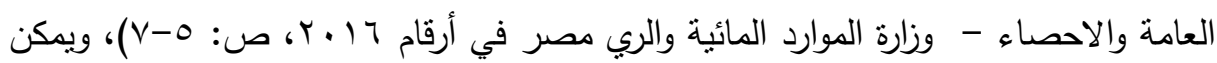

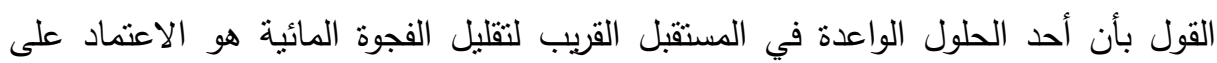

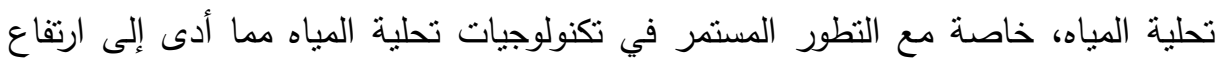
جودة المياه الناتجة وصلاحيتها للشرب، ويساعد الموقع الجغرافي لمصر على توافر مصدر

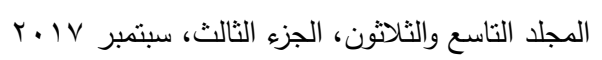


المياه المالحة حيث يحدها البحر الأحمر بامتداد حدودها شرقاً والبحر الأبيض بامتداد حدودها

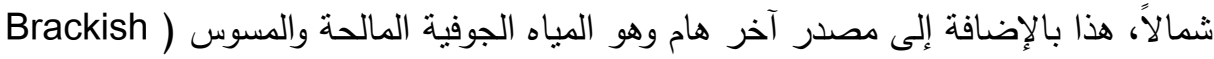
water والتي يمكن أن نساهم كمصدر للتحلية وذلك لسد احتياجات المجتمعات المحلية في

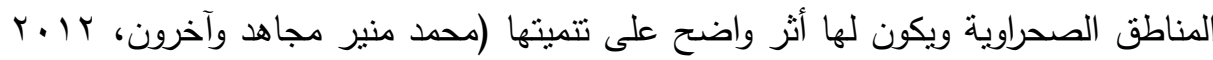

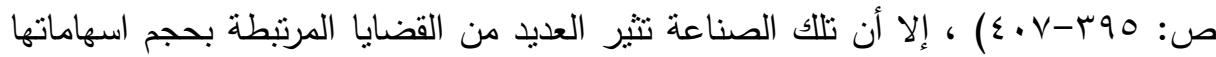
كأحد الموارد غير التقليدية لمواجهة الثح المائي بالأراضي الجديدة، وذلك في ظل التكلفة المرتقعة التي تقوم عليها، خاصة فيما يتعلق بالاستهلاك الكثيف للطاقة والتي تعتبر أحد

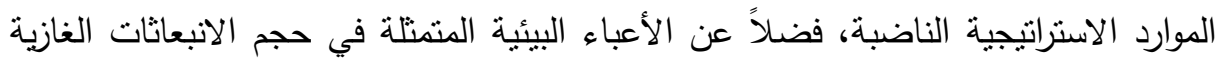

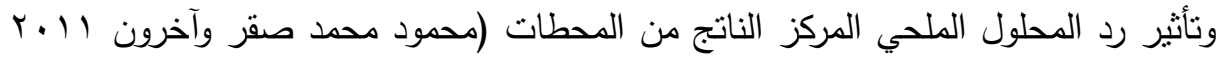
ص: 10-11)، لذا نتمنل المشكلة في كيفية الموازنة بين توفير المياه العذبة المحلاة تلبية

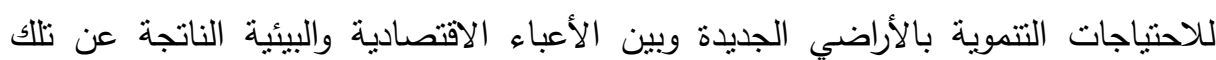

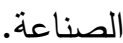

وفي ضوء ما سبق يمكن صياغة مشكلة البحث من خلال التساؤلات التالية:

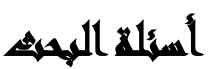

• ما مجموعة العوامل المؤثرة على تكلفة انتاج وحدة المياه المحلاة من الآبار عالية الملوحة؟

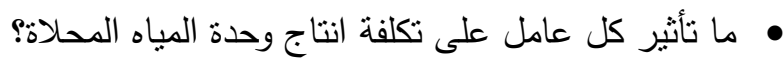

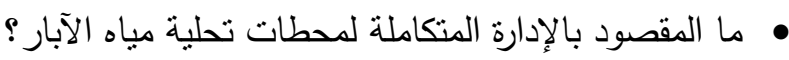

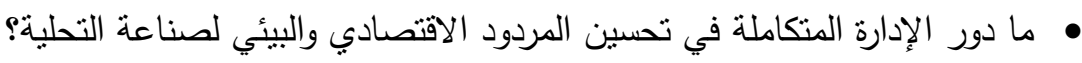

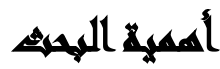

ترجع أهمية البحث إلي كونه احد الدراسات التطبيقية التي تُساهم في دعم واتخاذ القرار الخاص بالتتمية المستدامة للعديد من المناطق الصحراوية، وذلك عن طريق تحديد القيم التقريبية المتوقعة لتكلفة وحدة المياه الدحلاة تحت ظروف التشغيل المختلفة بالأراضي 
الصحراوية، فضلاً عن التقييم الاقتصادي لنموذج الإدارة المتكاملة لمحطة تحلية مياه مصغرة تعمل بالطاقة المتجددة بمنطقة الثلاتين.

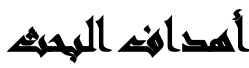

يستهدف البحث بصورة رئيسية دراسة اقتصاديات إدارة المحطات المصغرة لتحلية مياه الآبار عالية الملوحة بصورة متكاملة تحقيقاً لأغراض التتمية المستدامة بالأراضي الصحراوية، وذلك من خلال تحقيق الأهداف التالية: 1-تحديد مجموعة المتغيرات المستقلة المؤثرة على تكلفة انتاج وحدة المياه المحلاة. r- بناء نماذج محاكاة لتقدير تكلفة وحدة المياه المحلاة بمعلومية مجموعة المتغيرات المستقلة

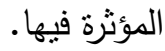

r-دراسة الوضع الراهن لتحلية المياه بمصر ، والتعرف على الطرق الحديثة لتحلية المياه بالاعتماد على الطاقة الثمسية. ع-دراسة آليات تطبيق أسلوب الإدارة المتكاملة لمحطات تحلية مياه الآبار عالية الملوحة. 0- التحليل المالي لنموذج تطبيق الإدارة المتكاملة لمحطة تحلية مياه بمدينة الثلاتين.

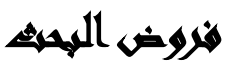

تتمثل فروض البحث في النقاط التالية: 1- توجد علاقة معنوية بين درجة ملوحة مياه المصدر وتكلفة انتاج وحدة المياه المحلاة. r-توجد علاقة معنوية بين درجة حرارة مياه المصدر وتكلفة انتاج وحدة المياه المحلاة. ب-توجد علاقة معنوية بين نسبة تركيز الأملاح في المياه المنتجة وتكلفة انتاج وحدة المياه. ع-توجد علاقة معنوية بين السعة الانتاجية لمحطة التحلية وتكلفة انتاج وحدة المياه المحلاة.

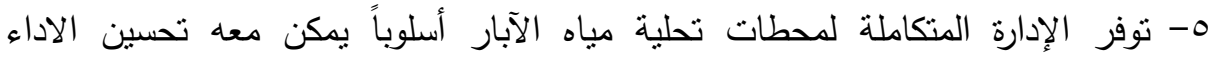
الاقتصادي والبيئي لمشروعات تحلية المياه بالأراضي الصحراهية الإدارية. 


\section{مسور الهمبه}

الحد الزمنى: رصد وحساب التكاليف الرأسمالية والتكاليف المتغيرة كذلك العائد لـشروع

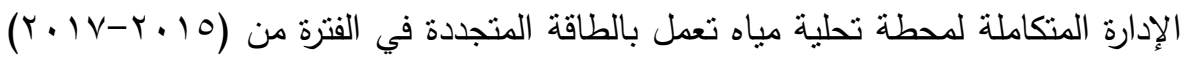
بمنطقة الدراسة. الحد المكاني: مدينة الثلاثين - محافظة البحر الأحمر - جمهورية مصر العربية

\section{التراسايت الساريه}

في دراسة للوكالة الدولية للطاقة الأرية International Atomic Energy عن اقتصاديات تحلية مياه البحر باستخدام برنامج DEEPcy

•أهم الأهداف: اجراء شامل لمقارنة تكاليف التحلية باستخدام مصادر الطاقة النووية والوقود

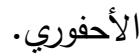

•أهم النتائج: إن تكاليف عمليات تحلية المياه بالاعتماد على الوقود النووي كانت أقل بصورة

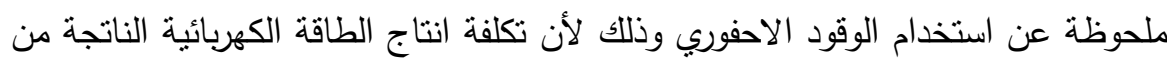

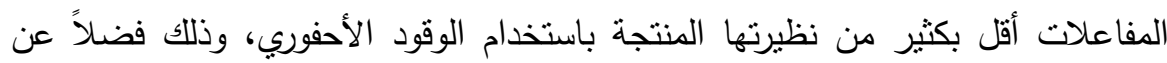

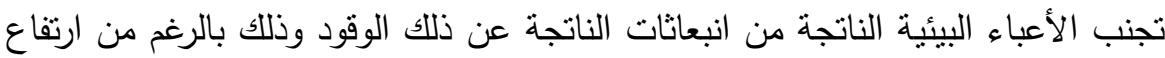

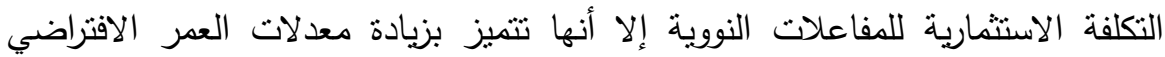
بصورة كبيرة عن المولدات المستخدمة في انتاج الكهرباء من الوقود الأحفوري. •أهم التوصيات: التوسع في انتاج المفاعلات صغيرة الحجم والتي تتميز بانخفاض تكلفتها الاستثمارية ومناسبتها لتشغيل محطات التحلية صغيرة الحجم بالعديد من الأماكن النائية. 
وفي دراسة لـHafez, S. El-Manharawy عن اقتصاديات تحلية المياه باستخدام تقنية التناضح العكسي في محافظة البحر الأحمر، تبين ما يلي: •أهم الأهداف: تقدير التكاليف الرأسمالية وتكاليف النتغيل والصيانة لعطليات تحلية المياه باستخدام تقنية التتاضح العكسي.

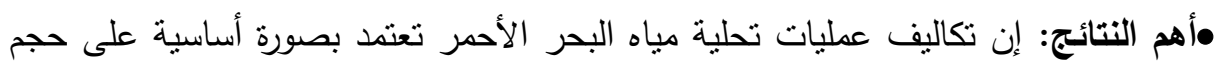

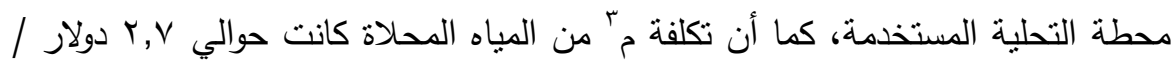

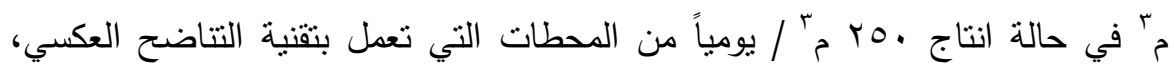

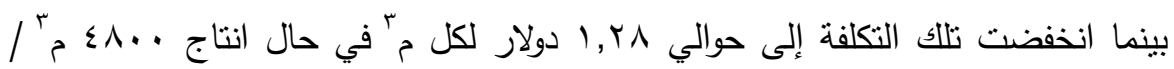

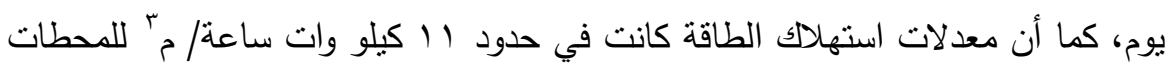

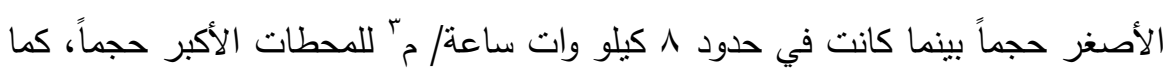

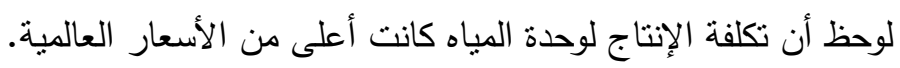

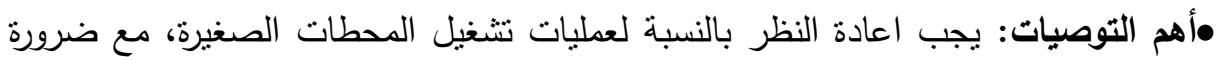

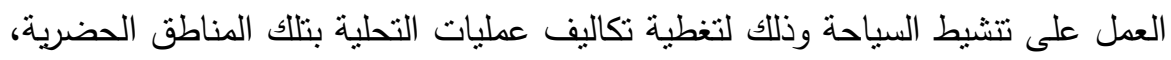

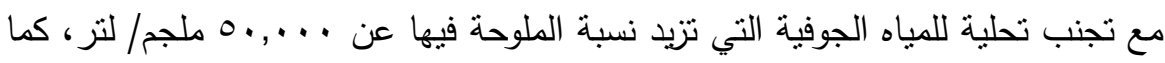

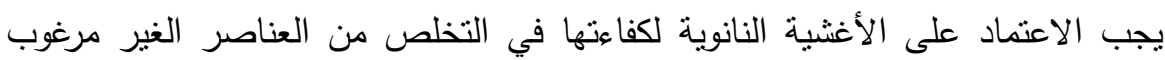
فيها.

وقد قام كلاً من Asmerom M. Gilau \& Mitchell J. Small بتصميم نظام لقياس فاعلية تكلفة انتاج المياه المحلاة في الدول النامية وأوضحت

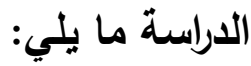
•أهم الأهداف: استخدام برنامج ROSA لتصميم مجموعة نماذج ليحطات تحلية ذات سعات التمات

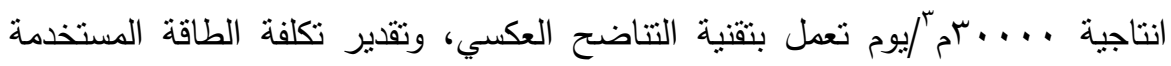
والتكلفة الاجمالية لوحدة المياه بالاعتماد على الطاقة الثمسية وطاقة الرياح. 
•أهم النتائج: أوضحت نتائج الدراسة أن تكلفة الطاقة اللازمة لإنتاج وحدة المياه المحلاة

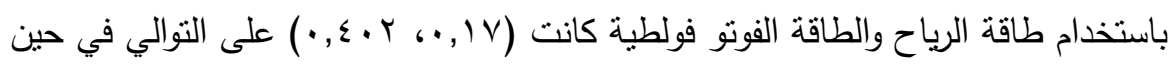

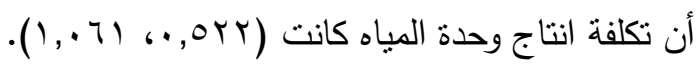

أما G.V. Reddy, N.Ghaffour فقد قاما بإجراء دراسة عن طرق تقدير تكاليف تحلية المياه بالاعتماد على التقتيات التجارية لتحلية المياه وإتضح ما

•أهم الأهداف: يهدف هذا البحث إلى تقديم نظرة عامة عن التكاليف الخاصة بعطليات التحلية

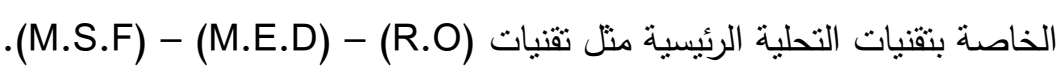
•أهم النتائج: تنين من نتيجة الدراسة الخاصة بتقنيات التحلية المتعددة أن التكاليف وأوجه الصرف ليست ثابتة أو محددة، ولكنه من المؤكد أن تكاليف عمليات التحلية قد تتاقصت بلته بصورة واضحة خلال العقود الثلاثة السابقة، كما أن تقنيات التحلية وبصفة خاصة تنفة تنبية التتاضح العكسي (R.O) سوف نتافس المصادر التقليدية في القريب العاجل، كما أن هنالك علاقة ارتباط قوية بين تخفيض تكلفة التحلية وزيادة الطاقة الانتاجية من عمليات التحلية.

في حين قامت Dalia E. Abo Zaid بإجراء تحليل اقتصادي لمحطة تحلية مياه مصغرة ومتنقلة تعمل بتقتية التناضح العكسي وتدار بالطاقة الثمسية تحت ظروف الساحل الثمالي الغربي بمصر واتضح من خلال تلك الاراسة ما •أهم الأهداف: دراسة الآثار الاقتصادية والاجتماعية لمحطة تحلية مياه متتقلة تعمل بتقنية التتاضح العكسي اعتماداً على الطاقة الثمسية.

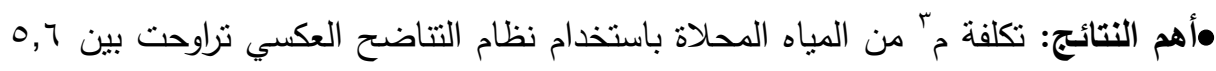

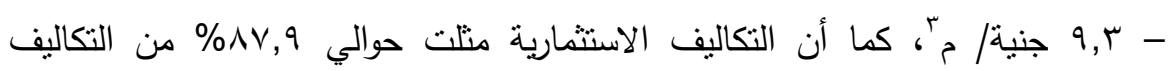

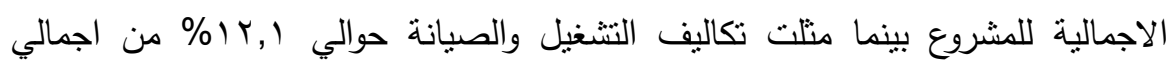


التكاليف، كما أمكن تخفيض تكلفة وحدة المباه بصورة واضحة بعد استخدام بطارية، وحتى عند هذا المستوى من التكلفة فإن هذا النظام قادر على توفير الكميات الضرورية من المياه

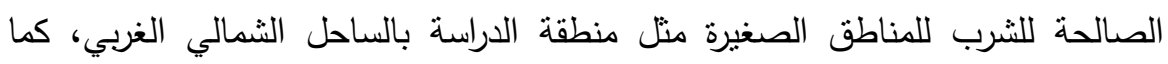

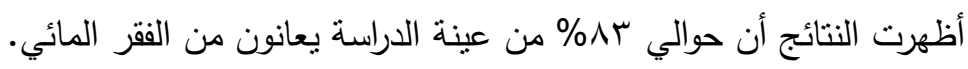

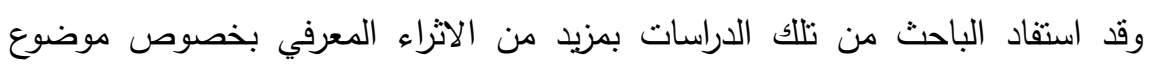
الدراسة وعلى سبيل المثال فقد ترسخت فكرة أن تكلفة وحدة المياه المحلاة تتتاقص كل فترة التراه وذلك بسبب التطور التقني في عمليات التحلية، كما أن إدارة الموارد المائية يجب أن تتم وفقاً لمبادئ الإدارة المتكاملة سواء بالنسبة للمورد الواحد أو للموارد المتعددة فيما بينها.

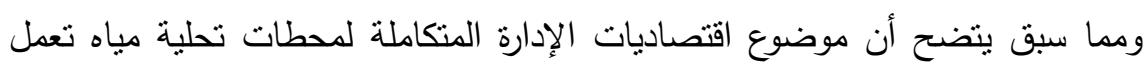
بالطاقة المتجددة في الأراضي الجديدة هو محل دراسة ويحتاج لمزيد من التحليل للوصول

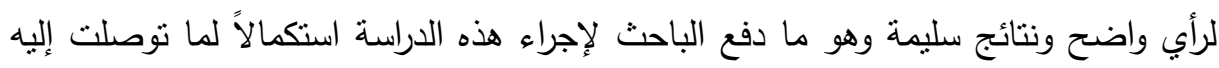

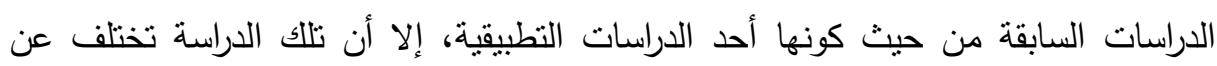
السابقة فيما يلي: أولاً في المجال البحثي: حيث تبرز الجوانب الاقتصادية والبيئية لتحلية المياه باستخدام الطاقة المتجددة، وذلك اعتماداً على أسلوب الادارة المتكاملة والذي يمكن من تحقيق أهداف التهاب التنمية من خلال وجهة النظر القومية والمنتكلة في توفير المياه اللازمة للشرب والزراعة وتتمية المجتمع الصحراوي مع الالتزام بمبادئ التتمية المستدامة وكذللك تعظيم العائد من وحدة المياه المنتجة والعمل على وضع استراتيجية لتخفيض التكاليف الخاصة بعمليات التحلية، وتدنية

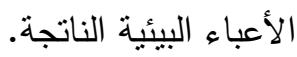
ثانياً: في المجال التطبيقي: حيث أن تلك الدراسة سوف تتم على مشروع تحلية المياه باستخدام محطات تحلية تعمل بتقنية التتاضح العكسي (R.O) وذللك اعتماداً على الطاقة الثمسية كمصدر للحصول على الطاقة اللازمة لنتغيل المشروع في منطقة الثلاتين بحيث تتم إدارة هذا المشروع وفقاً لحزمة من الاجراءات المنبثقة من مبادئ الإدارة المتكاملة للموارد 


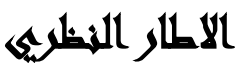

أولاً: الوضع الحالي والاحتياجات المستقبلية من مياه التحلية المياه في مصر: تبلغ الطاقة الإنتاجية الحالية لمصر من المياه المحلاة حوالي (0.1) مليار م سنوياً

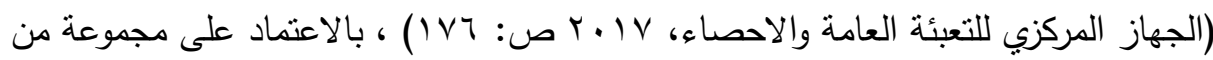
محطات تحلية المياه الموزعة على مستوى الجمهورية، إلا أن الإنتاج الحالي من المياه المحلاة لا يتتاسب مع الإمكانيات الهائلة المتوفرة، لذا فقد قامت ولئ وزارة الري والموارد المائية

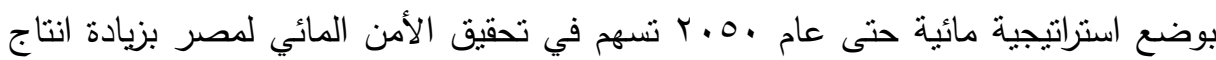

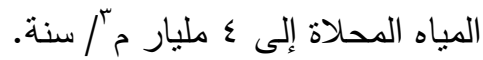
ثانياً: التقتيات المستخدمة في محطات إنتاج المياه المحلاة: تهدف تحلية المياه إلى إزالة أو خفض الأملاح الذائبة بمياه البحر أو المياه الجوفية أو

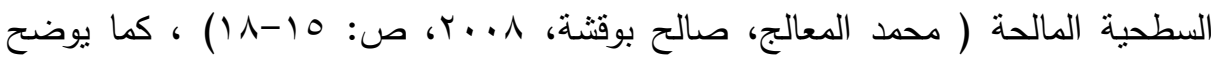
الثكل رقم (1) الطرق المختلفة للنحلية والتي تتم إما بتغيير الحالة الطبيعية للمياه بتحويلها من سائل إلى بخار يكثة فيما بعد وهو ما يعرف بالطرق الحرارية، والتي تشمل تقنيات

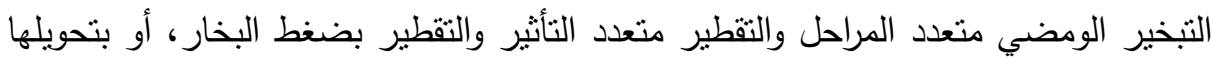
من الحالة السائلة إلى الحالة الصلبة وهو ما يعرف بطريقة التجميد والتي يتم فيها غسل الأملاح بعد تسييل المباه، وهنالك بعض تقنيات التحلية التي تعمل دون تغيير الحالة الطبيعية

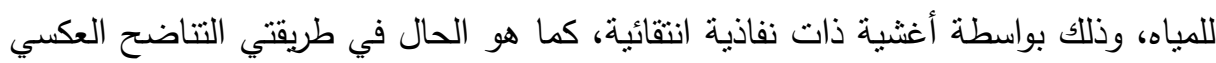
والفرز الكهربائي، كما يمكن استخدام الخواص الانتقالية للأيونات في إزالة الملوحة كطريقة

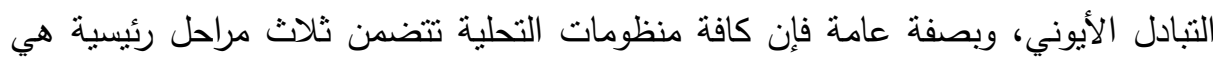
المعالجة المبدئية، وحدة التحلية، والمعالجة النهائية، بحيث تهدف المعالجة المبدئية إلى تهيئة المباه المالحة لعملية التحلية، والتي تتضمن عدداً من عمليات المعالجة الفيزيائية والكيميائية المستخدمة، بينما تهدف المعالجة النهائية إلى جعل المياه المحلاة مناسبة للاستخدامات المقصودة ويتم تحديد المعالجات النهائية المطلوبة وفقاً لذلك، "وتنتأثر تكنولوجيا التتاضح

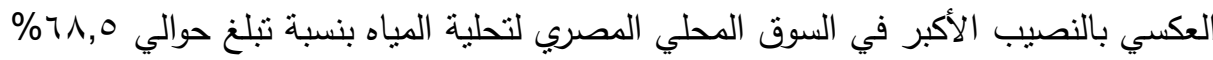


من انتاج المياه المحلاة، تليها تقنية التبخر الومضي بنسبة V,Y/\%" ( وحدة البحوث

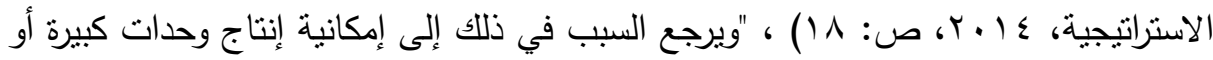

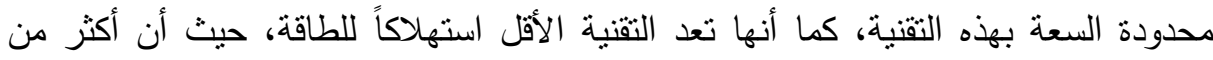
تلثي الطاقة الإنتاجية للمياه المحلاة في مصر تستخدم في قطاعات السياحة والصناعة

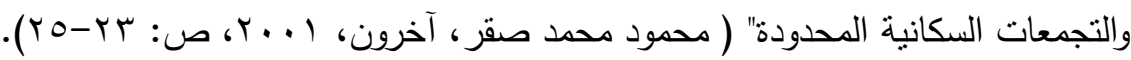

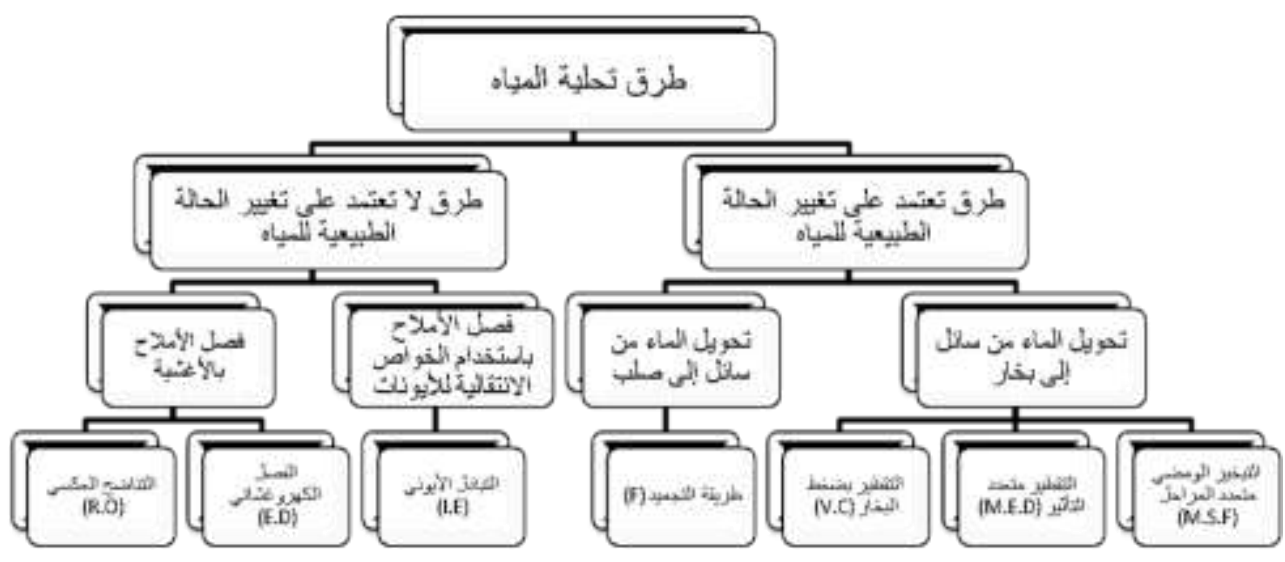

شكل رقم ( ) ): شكل توضيحي لطرق تحلية المياه

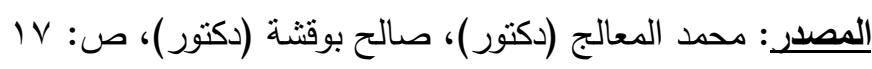
ثالثاً: استخدامات الطاقة المتجددة في تحلية المياه: توفر التحلية باستخدام الطاقة المتجددة طريقة مستدامة لإنتاج المياه العذبة وبصفة خاصة في تلك الأماكن النائية بالأرضي الصحراوية والتي تفتقر إلى امدادات المياه والكهرباء عن طريق الثبكات العامة، لذا فإن الاستفادة من المصادر الطبيعية المتاحة بالموقع المراد تتميته قد يكون حلاً فعالاً لتأمين الاحتباجات اللازمة من الطاقة بصفة خاصنة فئهة في ظل الانخفاض المستمر في تكلفة وحدة الطاقة المنتجة من تلك المصادر ، "وبالرغم من تلك المزايا إلا أن نسبة المياه المحلاة باستخدام الطاقة المتجددة لا تتعدى / \% من اجمالي انتاج المياه 
المحلاة على مستوى العالم، حيث تستحوذ تقنية (RO) على ما يقرب من بr\% من انتاج

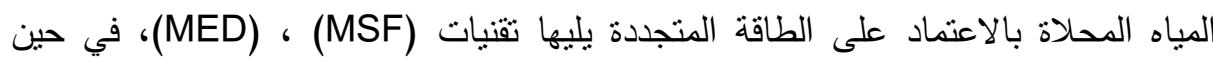

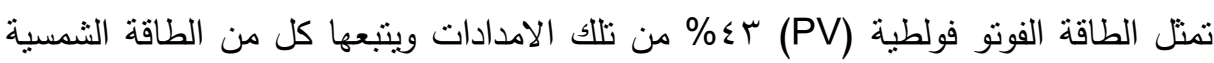

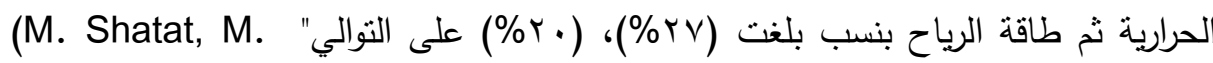

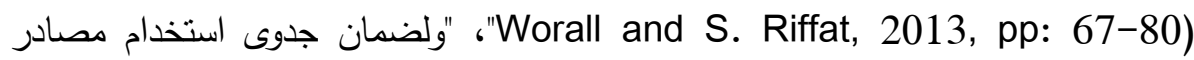

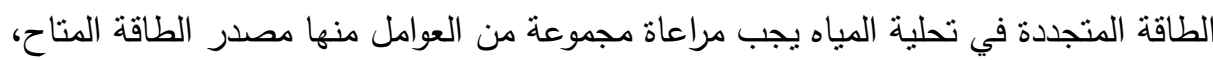
خصائص الموقع، درجة ملوحة مياه المصدر والسعة الانتاجية لمحطة التحلية، "حيث أن أن

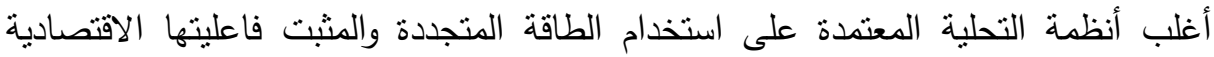

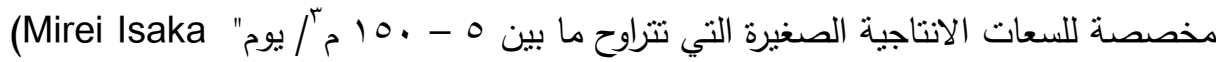
أن Giorgio Simbolotti, 2013, pp: 122) أفضل بدائل أنظمة تحلية المياه باستخدام الطاقة المتجددة وأوسعها انتتاراً خاصة في الأماكن

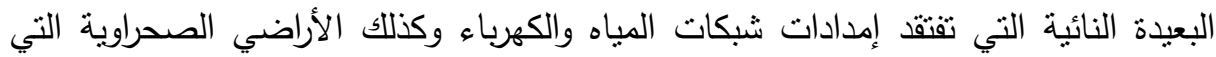
تتميز بارتفاع معدلات سطوع الثمس، كما أنه يعد من الأنظمة المجدية اقتصادياً خاصة عنداه ولداه

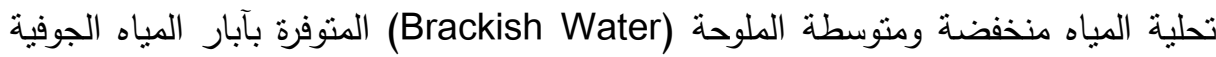
بالصحاري، وهو مناسب كذلك للسعات الانتاجية الصغيرة لإمكانية توصيل التيار المباشر

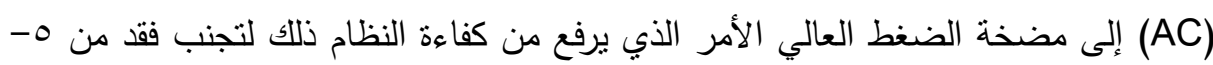

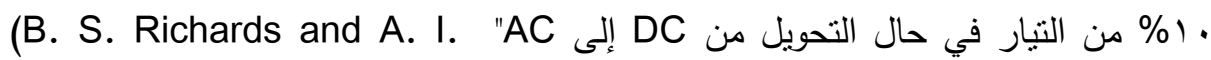
.Schafer, 2010, p: 363) رابعاً: نموذج الإدارة المتكاملة لمشروع تحلية المياه بالأراضي الجديدة: يرتكز فكر الإدارة المتكاملة لمشروع تحلية المياه بالأراضي الجديدة على الاستخدام

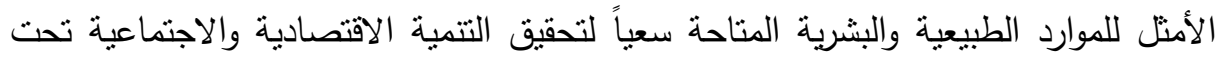
مظلة الاستدامة الايكولوجية بتلك الأراضي وذلك عن طريق اعتماد مجموعة مقترحة من الحزم التقنية المتكاملة لإنتاج المياه المحلاة، انتاج الأسماك، الانتاج النباتي والانتاج الحيواني النافي كالتالي: - n 
1-انتاج المياه المحلاة لنوفير احتياجات المجتمعات الصحراوية من مياه الثرب باستخدام

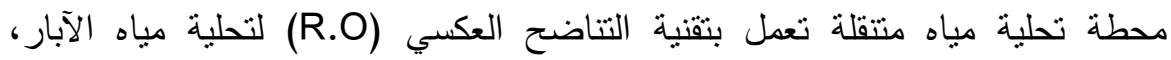
بالاعتماد على الطاقة الثمسية الفوتو فولطية (P.V) كمصدر متجدد لتوفير الكهرباء

$$
\text { اللازمة لها. }
$$

ץ-استخدام المحلول الملحي الناتج من عملية تحلية المياه بعد معالجته لاستزراع بعض أنواع

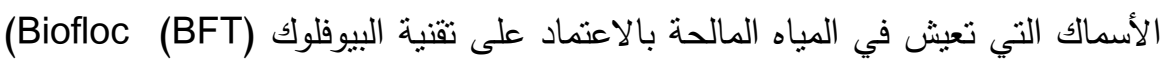
Technology) القاحلة وشبه القاحلة في جمهورية مصر العربية. r-زراعة المحاصيل العلفية المتحملة للملوحة باستخدام المياه الناتجة من الأحواض السمكية والمشبعة بالأسمدة العضوية اللازمة لتغذية النبات. ع-نظوير انتاج الثروة الحيوانية بنلك المناطق عن طريق تصنيع الغذاء اللازم لها من المحاصيل العلفية.

\section{الطريقة البحثية ومصادر البيانات:}

تم اعداد ما يسمى بدليل المقابلة، واعتمد البحث على المنهج الاستقرائي (الاستعراض

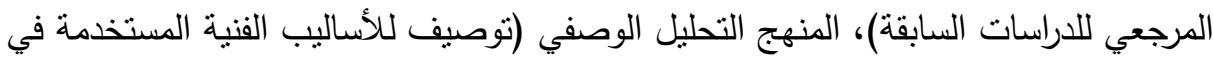
التحلية، استخدامات الطاقة المتجددة في التحلية، نموذج الإدارة المتكاملة لمشروع تحلية مياه الآبار)، المنهج القياسي الكمي (عمل نماذج محاكاة باستخدام البرنامج الاحصائي DEEP

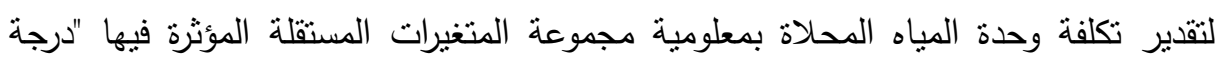
ملوحة مياه المصدر ، درجة ملوحة المياه المنتجة، درجة حرارة مياه المصدر ، السعة الانتاجية لمحطة التحلية"، وتقدير العلاقة الرياضية بالاعتماد على اسلوب الاتحدار الخطي البسيط دله وأخيراً استخدام أسلوب التحليل المالي لنموذج مشروع الإدارة المتكاملة لمحطة تحلية مياه

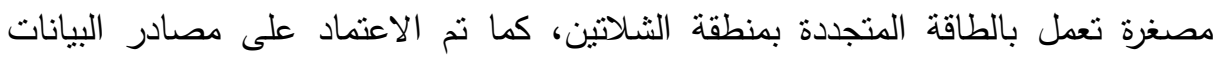
الثانوية المنشورة وغير المنشورة التي تصدرها بعض الجهات المعنية مثل الهيئات الحكومية

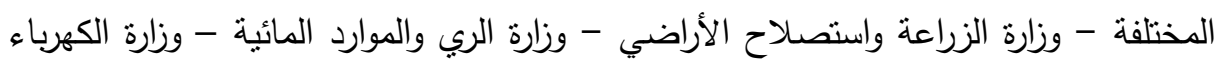

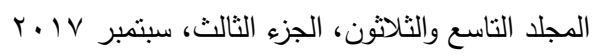


والطاقة المتجددة - مركز بحوث الصحراء - مركز التميز المصري لأبحاث التحلية (EDRC) للطاقة (IEA)- البنك الدولي - الجهاز المركزي للتعبئة العامة والاحصاء - محافظة البحر الأحمر، وكذلك من خلا اجراء المقابلات الثخصية مع المسئولين عن محطات التحلية المصغرة بالأراضي الصحراوية للحصول على البيانات الميدانية الخاصة بالتتفيذ الفعلي لمبادرة حل المشاكل الضاغطة في المجتمعات الصحراوية بالاعتماد على تطوير محطات تحلية مياه

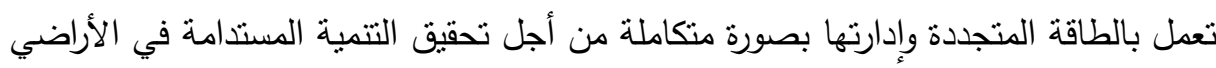
الصحراوية. عينة الدراسة الميدانية: نم عمل ما يسمى بدليل المقابلة وباستخدام أسلوب الحصر الثامل تم دراسة نأثثر مجموعة المتغيرات المستقلة ذات الأثر المباشر على تلى بكلفة وحدة المياه المحلاة

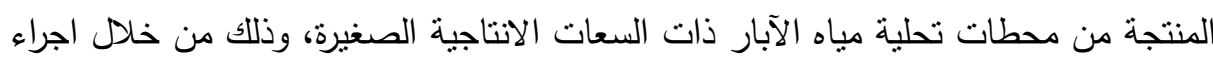
المقابلات مع المسئولين عن نشغيل محطات التحلية المصغرة بالأراضي الصحراوية كما تم دراسة النطبيق العملي لنموذج الإدارة المتكاملة لمحطة تحلية مصغرة تعمل بالطاقة المتجددة في منطقة الثناتنين.

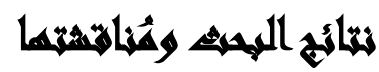

أولاً: التحليل الاقتصادي لتكلفة وحدة المياه المحلاة: 1-العوامل المؤثرة على تكلفة انتاج وحدة المياه المحلاة:

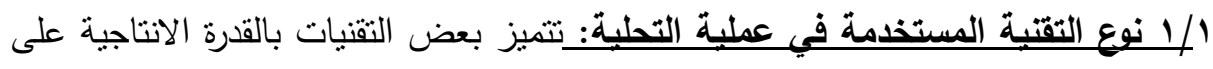

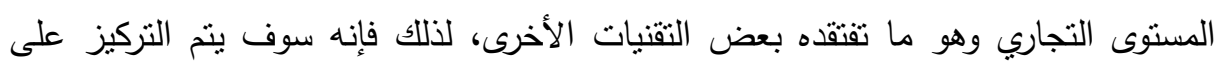

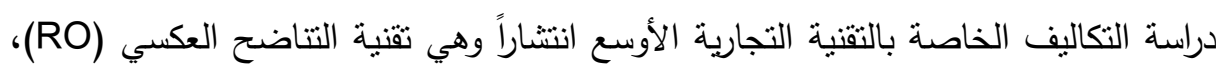

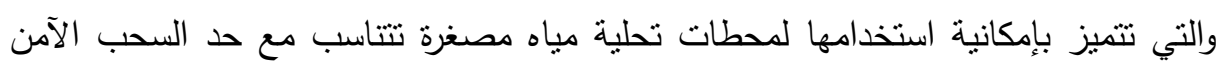
من الآبار ، اضافة إلى كونها التقنية الأقل استهلاكاً للطاقة. 
r/I مصدر الطاقة المستخدم: تتأثر تكلفة التحلية بمصدر الطاقة المستخدم نظراً لكونها من الصناعات ذات الاستهلاك الكثيف للطاقة، لذا فإنه سوف يتم دراسة مدى تأتز تكلفة الوحدة من المياه المحلاة بتغير نوع الوقود المستخدم من المصادر المختلفة (تقليدية - جديدة -

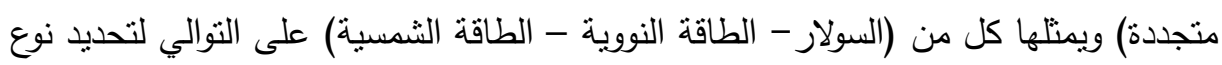
الوقود الأنسب لعملية التحلية من حيث مدى توافره بموقع محطة التحلية وكذا استدامته.

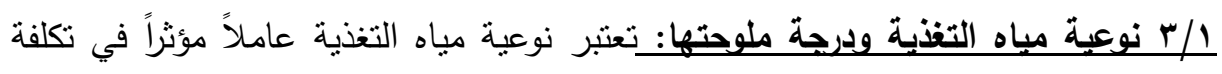

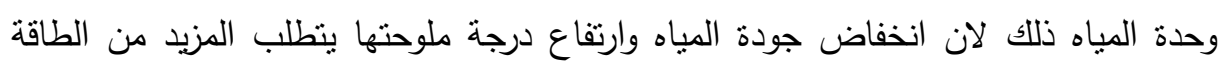
والمواد الكيميائية المعالجة.

فكل كودة المياه المنتجة:حيث أن إنتاج مياه للثرب يختلف نوعاً عن انتاج مياه للزراعة، فكل له المواصفات الخاصة به وذلك وفقاً لنسب الأملاح الذائبة المسموح به في نوعية المياه المياه

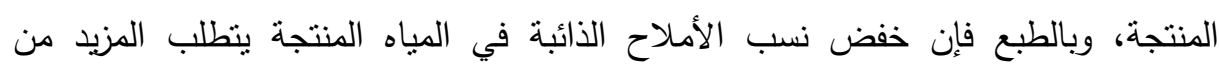
عمليات المعالجة وكذلك الطاقة المستخدمة.

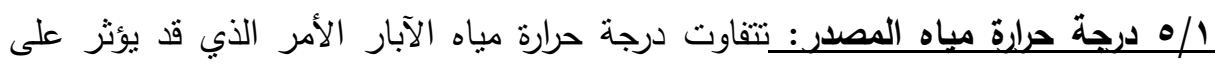
معدلات استهلاك المواد الكيميائية المستخدمة في عملية معالجة المياه وكذلك معدلات دلات داه استهلاك الطاقة المستخدمة في التحلية. 7/1 السعة الانتاجية لمحطة التحلية: تتأثر تكلفة وحدة المياه المحلاة بالقدرة الانتاجية

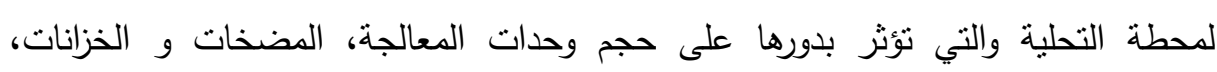

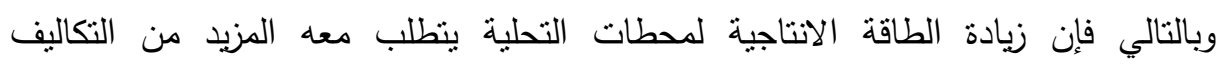
الرأسمالية، وتكاليف العمالة اللازمة للتشغيل وعلى الرغم من ذلك فإدانه الطنه عند تحليل فاعلية التكلفة فإن تكلفة انتاج الوحدة ينخفض مع زيادة حجم الانتاج. r r r عناصر التكاليف الخاصة بمحطات تحلية المياه

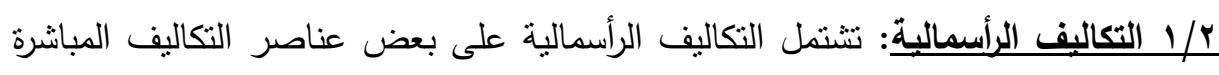
وأخرى غير المباشرة وهي كالتالي: 


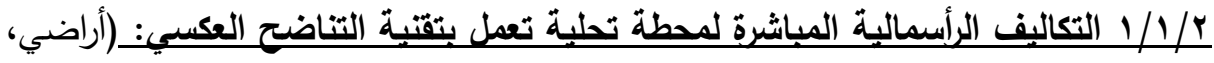

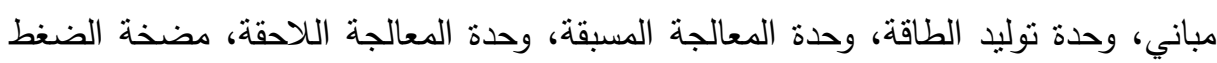

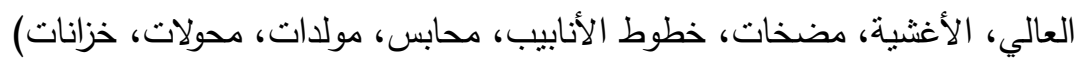

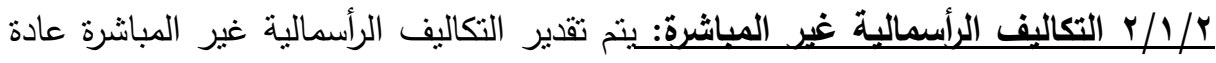
كنسب مئوية من اجمالي التكاليف الرأسمالية المباشرة، وقد تشمل تكاليف شحن وتأئرة الأجهزة والمعدات، تكاليف بناء المعامل والمباني الإدارية والمكاتب وأجهزة الأمن الصناعي.

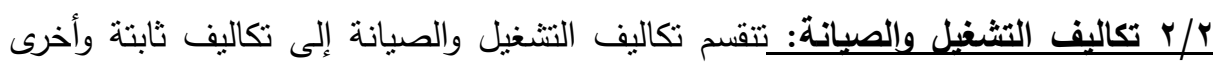

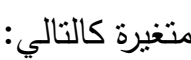
1/r/r/r/ التكاليف الثابتة: تشتمل التكاليف الثابتة على تكلفة التأمين، ورواتب الإداريين،...

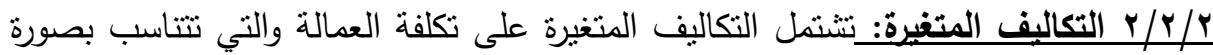
طردية مع حجم الطاقة الانتاجية للمحطة، وكذلك الطاقة حيث تختلف حجم الطاقة المستخدمة باختلاف نوع التقنية المستخدمة في التحلية كما تتأثر تكلفة الطاقة بمصدر الطاقة المستخدم ومدى توافره، وأيضاً الكيماويات والتي تتأثز تكلفتها بدرجة ملوحة مياه المصدر ودرجة حراتهن حرارتها وكذلك درجة جودة المباه المنتجة وذلك بناءً على كمية ونوعية الكيماويات المستخدمة، وأخيراً الصيانة والتي تتأثز أيضاً بنوعية مياه المصدر ونسب نركيز الأملاح الذائبة بها والتي لها دور رئيسي في معدل تغيير الأغشية. r- نموذج تقدير تكلفة وحدة المياه المحلاة: يستهدف هذا الجزء من البحث تقدير تكلفة وحدة المياه المحلاة من محطات التحلية تعمل بتقنية التتاضح العكسي (RO)، عن طريق بناء نماذج محاكاة لتقدير تكلفة وحدة المياه المحلاة بمعلومية مجموعة المتغيرات المستقلة المؤثرة فيها باستخدام أحد البرامج الاحصائية المختصة بتقييم وتحليل هيكل التكاليف الخاص وداه

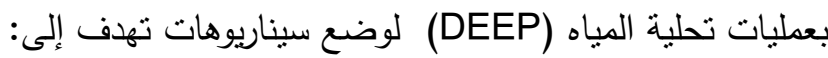

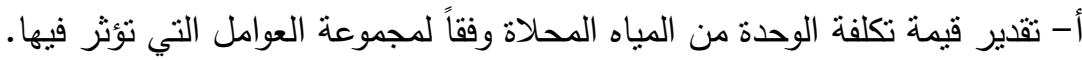

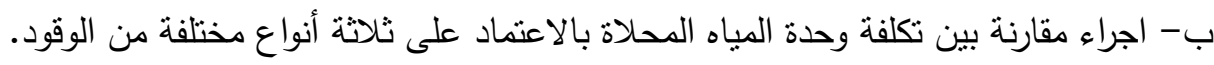


ج - تحديد الصورة الرياضية للعلاقة التي تربط كل متغير مستقل بالمتغير التابع (تكلفة وحدة

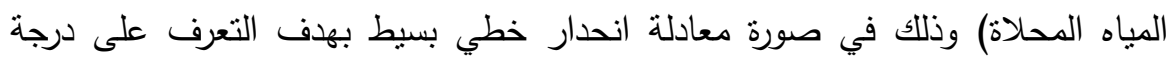
تأثثر كل عامل على تكلفة انتاج وحدة المياه المحلاة.

1-قياس تأثير اختلاف درجة ملوحة مياه المصدر على تكلفة وحدة المياه المحلاة:

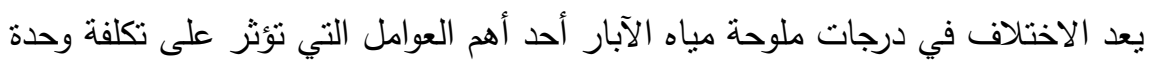
المياه المحلاة، وهو ما يتضح من خلال جدول رقم (1) حيث تم تقدير تأثنير اختلاف درجة

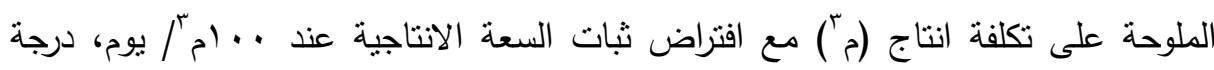

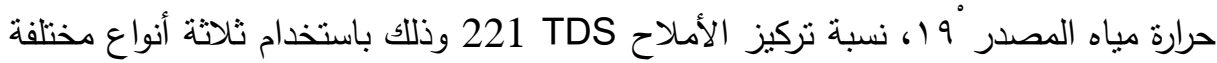
من الوقود، بغرض تحليل هيكل التكلفة لوحدة المياه المنتجة وتحديد أثر زيادة درجات ملوحة مياه المصدر على عناصر التكلفة المختلفة.

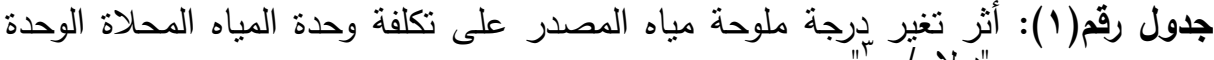

\begin{tabular}{|c|c|c|c|}
\hline \multicolumn{3}{|c|}{ 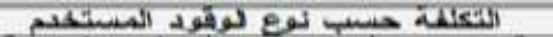 } & \multirow{2}{*}{ درية ملقحة ميا • الفصدر (Ppm) } \\
\hline 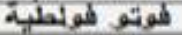 & Sare & $\operatorname{seg} 2029$ & \\
\hline$\cdot$. Ara & $\cdot, \lambda \cdot \theta$ &., 791 & Y... \\
\hline$\cdot A \mathbf{A}$ & $\because, A \cdot A$ &., $77 i$ & E... \\
\hline., $17 v$ & $\because, A F r$ & .719 & $7+\cdots$ \\
\hline 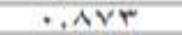 & $\because$ ATA & $.79 \%$ & A. . . \\
\hline *AA & $\because A \geq 0$ & $.74 \mathrm{~V}$ & $1 \cdots$ \\
\hline 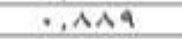 & .,AOY & $\cdot, \mathrm{V} \cdot \mathrm{r}$ & $17 \ldots$ \\
\hline 0,147 & .,NOA & $\because V, V$ & $12 \ldots$ \\
\hline. .977 &., $4 \mathrm{ra}$ & . vVr & $17 \ldots$ \\
\hline $.9 V \mathrm{E}$ & $0.9 \times 7$ &. $\mathrm{VVV}$ & 今心... \\
\hline$\cdot .9 \wedge r$ & $0,4 t i$ & $\cdot$ var & Y.... \\
\hline $0.44 \mathrm{r}$ & 0.908 & $\therefore \vee \wedge \vee$ & YY... \\
\hline $1, \ldots r$ & $0.47 \mathrm{r}$ &,$V A r$ & $\mathrm{Y} i \ldots$ \\
\hline $1, \ldots r$ & $04 \mathrm{VI}$ & $\because v a v$ & r7... \\
\hline $1 . . r r$ & $.4 \wedge r$ & $\therefore A \cdot Y$ & YA... \\
\hline $1, . \times 7$ &., $49 r$ & 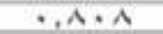 & $r \ldots$ \\
\hline $1, .0 r$ & $1, \ldots t$ & $\because \wedge i \wedge$ & $r r . .$. \\
\hline $1.7 \mathrm{~A}$ & $1, \ldots 1 \wedge$ & - AYY & $r i \ldots$ \\
\hline $1, .41$ & $1, .2$. & $\because \wedge E Y$ & $57 \ldots$ \\
\hline $1.1 . \mathrm{A}$ & 1.000 & - ANOI & rA... \\
\hline 1,179 & $1,+\vee 1$ & - 1009 & t... \\
\hline $1,1 \pm V$ & $1, .4 \mathrm{r}$ &., 174 & EY... \\
\hline $1,1 \vee \%$ & 1,110 & $\cdot A \Delta Y$ & 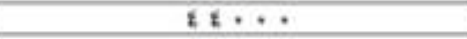 \\
\hline $1,7 \cdot 7$ & 1,120 & $\because 140$ & $27 \ldots$ \\
\hline $1, Y E Y$ & $1,1 \vee A$ &., $4 \cdot 1$ & $\varepsilon \wedge \ldots$ \\
\hline
\end{tabular}

المصدر: جمعت من عينة الدراسة الميدانية وحسبت من نتائج تحليل التكاليف باستخدام برنامج

(DEEP 5.1) 


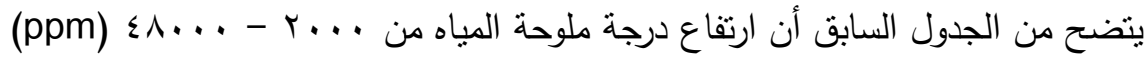

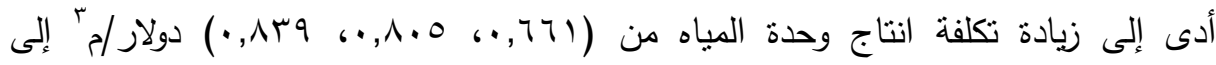

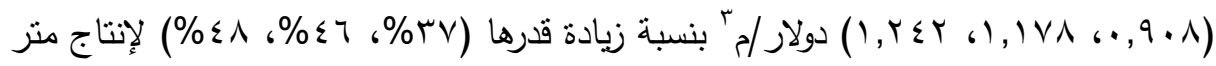

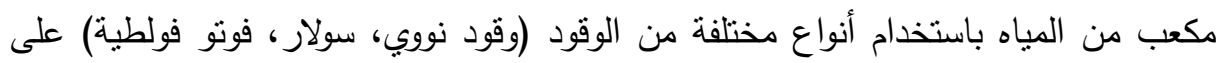

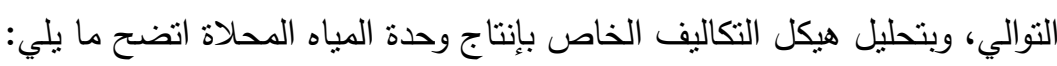
أ- - ترجع تلك الزيادة إلى تأثير ارتفاع درجة الملوحة على العناصر المختلفة لتكاليف الإنتاج

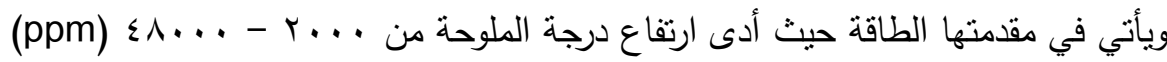

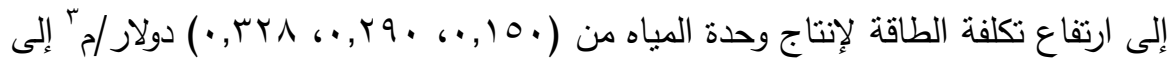

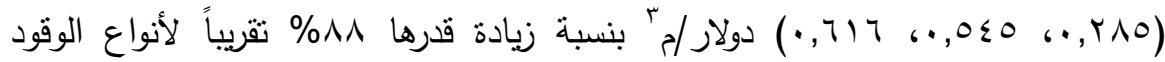
الثلاث.

ب- - ارتفاع اجمالي تكاليف استهلاك المواد الكيميائية، الأغشية والصيانة للوحدة المنتجة من

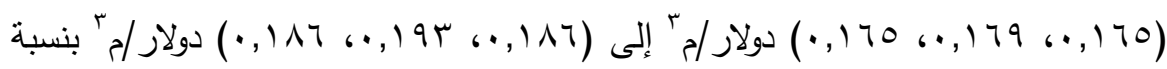

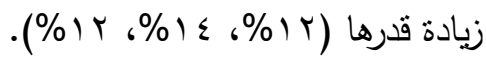

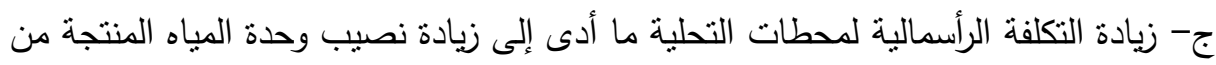

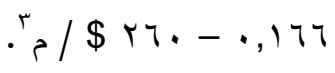

r-قياس تأثير اختلاف درجة حرارة مياه المصدر على تكلفة وحدة المياه المحلاة: تزيد

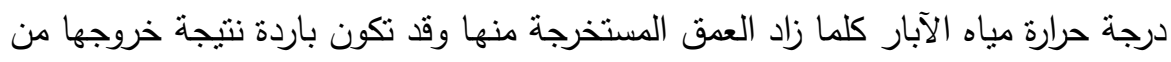
طبقات قريبة من سطح الأرض، وقد تم دراسة تأثثر ذلك الاختلاف في درجات الحرارة

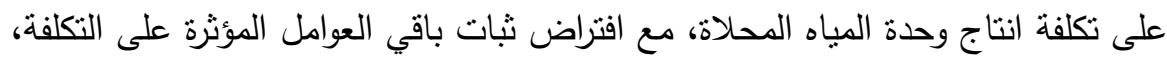

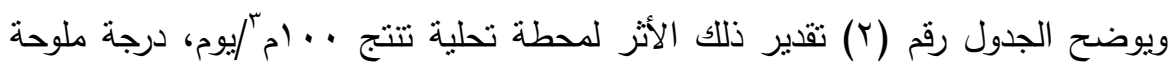
36000ppm 
عبير فرحات علي وآخرون

جدول رقم(ץ): أثز تغير درجة حرارة مياه المصدر على تكلفة وحدة المياه المحلاة الوحدة

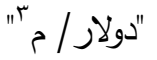

\begin{tabular}{|c|c|c|c|}
\hline \multicolumn{3}{|c|}{ التكعلفة حسبب نوع الوقوقد المستختح } & \multirow{2}{*}{ درجة حرارة مياه المصسر } \\
\hline فوتو فوالطِية (PV) & عنول & وققي نوبي (Nuclear) & \\
\hline $1,1,1$ & $1,0,7\}$ &., 100 & 10 \\
\hline 1,1, & 1,007 & $\cdot$, , & 17 \\
\hline $1,1, \varepsilon$ & 1,001 & 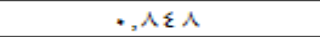 & 18 \\
\hline 1,098 & 1, . $\varepsilon 0$ & $\cdot, \lambda \varepsilon 0$ & 人 \\
\hline 1,091 & $1,0 \varepsilon$ & $\cdot$, , ᄉ $\varepsilon r$ & 19 \\
\hline 1,010 & $1, .50$ & $\cdot, \lambda \Gamma q$ & $r$. \\
\hline 1, , 人, & $1, .5$. & $\cdot, \lambda r \vee$ & ri \\
\hline $1,2 \times 0$ & $1, .47$ & $\cdot, \lambda 广 \varepsilon$ & $k t_{0}$ \\
\hline $1,+\sqrt{1}$ & $1,+4$ & $\cdot$, , NTr & $r r^{\prime}$ \\
\hline $1, * 7 \lambda$ & $1,+1 \mathrm{~V}$ & $\cdot, \lambda \Gamma \cdot$ & $r \varepsilon$ \\
\hline $1,+7 \varepsilon$ & $1,+1 \varepsilon$ & •,人イ人 & ro \\
\hline $1,+71$ & $1,+1$. & $\cdot$, Art & 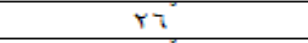 \\
\hline $1,0 \mathrm{~V}$ & $1, \cdots v$ & $\cdot, \lambda r \varepsilon$ & $r v^{\prime}$ \\
\hline $1,00 \varepsilon$ & $1, \ldots r$ & .,Arr & र人 \\
\hline $1,0,1$ & $1, \ldots$ & $\cdot$, , Аर. & $r q$ \\
\hline $1, \ldots q 9$ & $\cdot, 99 \mathrm{~V}$ & $\cdot$, 人,人 & r. \\
\hline $1,0 \leqslant 7$ & $\cdot, 990$ & $\theta, \lambda, \tau$ & 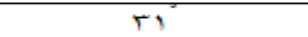 \\
\hline $1, * \varepsilon \varepsilon$ & $\cdot, 99 \mathrm{r}$ & $\cdot, \lambda, \varepsilon$ & $r t^{2}$ \\
\hline $1,+\varepsilon 1$ & $\cdot, 99$. & $\cdot, \lambda, r$ & $T$ \\
\hline 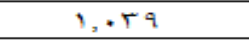 & $\cdot, 9 \wedge \vee$ & $\cdot, \lambda) \cdot$ & $T \varepsilon$ \\
\hline $1,+5 \mathrm{~V}$ & $\cdot, 9 \wedge 0$ & $\cdot, \lambda \cdot \wedge$ & ro \\
\hline $1,0.50$ & $\cdot, 9 \wedge T$ & $\cdot, \lambda \cdot \vee$ & $T$ \\
\hline$\ldots r \varepsilon$ & $\cdot, 9 \wedge \mathrm{r}$ & $\cdot, \lambda, 7$ & $r v$ \\
\hline
\end{tabular}

المصدر: جمعت من عينة الدراسة الميدانية وحسبت من نتائج تحليل هيكل تكاليف تحلية المياه

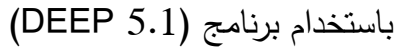
بدراسة النتائج الواردة بالجدول السابق تنين أن ارتفاع درجة حرارة مياه المصدر من 

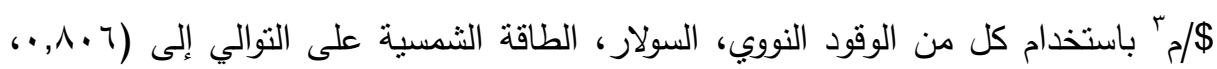

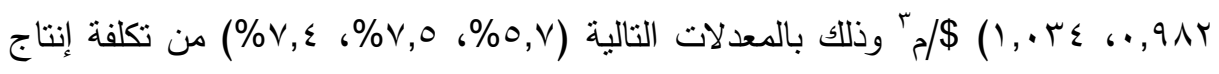

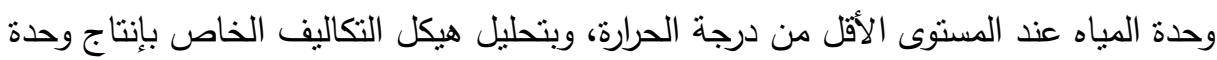

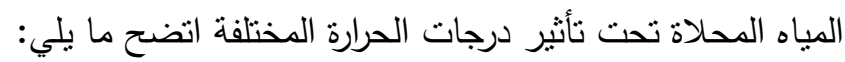


أ- تنتاقص تكلفة انتاج وحدة المياه المحلاة كلما ارتفعت درجة حرارة مياه المصدر وذلك نتيجة انخفاض كمية الطاقة اللازمة لإنتاج المنز المكعب من المياه المحلاة وبالتالي تتخفض تكلفة تلكك الطاقة عندما تكون درجة حرارة مياه المصدر 10 من لإنساه (

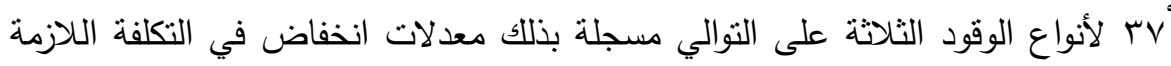

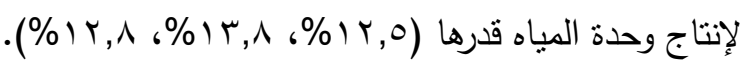

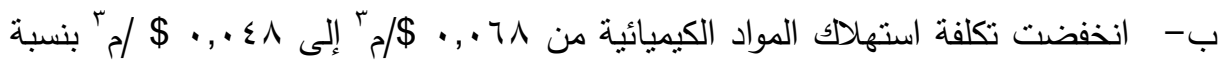

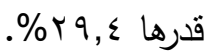

r-قياس أثر اختلاف نسبة تركيز الأملاح في المياه المنتجة على تكلفة وحدة المياه المحلاة: تعتمد المعالجة النهائية في تحلية المياه على الغرض من الاستخدام النهائي

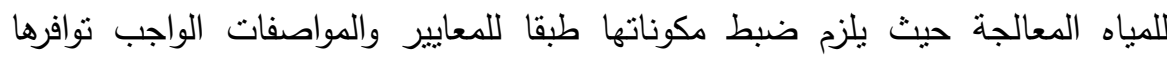
للاستخدامات المختلفة (شرب - ري - صناعة)، وبالتالي تختلف درجة المعالجة النهائية وفقاً لتلك المعايير، وبدراسة تأثير اختلاف درجة نركيز الأملاح في المياه المنتجة على رئى تكلفة انتاج وحدة المياه المحلاة، مع افتراض ثبات باقي العوامل المؤثرة على التكلفة،

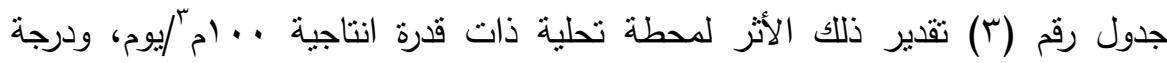

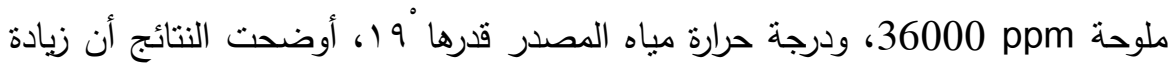

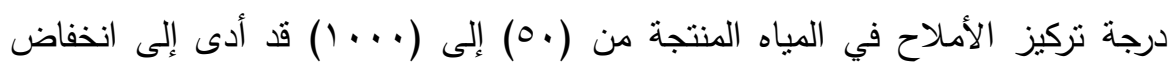

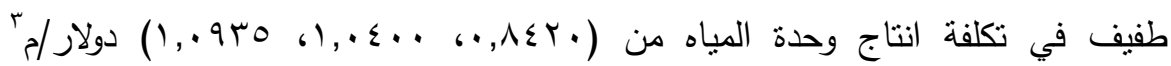

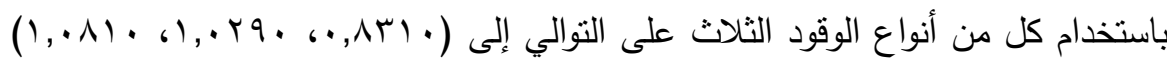

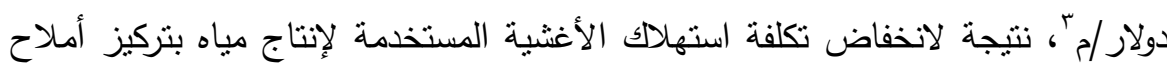


عبير فرحات علي وآخرون

جلول رقم(؛): أثز تغير نسبة تركيز الأملاح في المياه المنتجة على تكلفة وحدة المياه

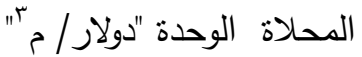

\begin{tabular}{|c|c|c|c|}
\hline \multicolumn{3}{|c|}{ 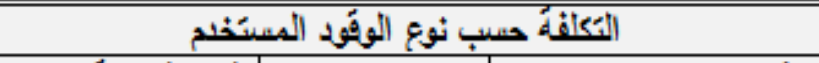 } & \multirow{2}{*}{ ثنب تركيز الأملاح } \\
\hline فونوتو فوإطبلة (PV) & عنولر. & وقُوحد نوبى (Nuclear) & \\
\hline 1,910 & $1,+\varepsilon Y O$ & $\cdot, \wedge \varepsilon \varepsilon 0$ & 0. \\
\hline $1,9+$. & $1, \varepsilon Y$, & $\cdot, \wedge \varepsilon \varepsilon$. & $1+$. \\
\hline 1,910 & $1,+\varepsilon, 0$ & , , ^हYO & 10. \\
\hline $1,91$. & $1, \varepsilon+$ & $\cdot, \wedge \varepsilon Y+$ & $r+$. \\
\hline $1,9,0$ & $1,+190$ &,,$\wedge \leqslant 10$ & ro. \\
\hline $1,9+\cdots$ & $1,+r q$ & $\cdot, \wedge \varepsilon 1$. & $\Gamma+$ \\
\hline 1,0190 & $1,+r 10$ & $,, \wedge \varepsilon, 0$ & ro. \\
\hline $1,+19$ & $1,+r V \cdot$ & $\cdot, \wedge 广 q$, & $\varepsilon \cdots$ \\
\hline 1,0110 & $1,+r 70$ & , , АT人० & \&o. \\
\hline $1,+\wedge \vee$ & $1,+r 7$ & $\cdot$, , К广人. & 0, \\
\hline $1, \times 170$ & 1,000 & , , ArVo & 00. \\
\hline $1,+17$ & $1,+10$. & $\cdot, \Lambda \Gamma V \cdot$ & $7+$ \\
\hline 1,0100 & 1. . Tro & ., 1 r OO & 70. \\
\hline 1,100, & $1,+\pi{ }^{\prime}$ & , , 1 ro. & $\mathrm{V}, \cdot$ \\
\hline $1,1,1<0$ & 1, TYO & $\cdot, \wedge \Gamma \varepsilon 0$ & vo. \\
\hline $1,+\wedge \varepsilon$ & $1,+r Y$, & $\cdot, \Lambda \Gamma \varepsilon$ & A.. \\
\hline $1,+1$ TO & $1,+10$ & , ,АTrO & 10. \\
\hline $1,+\Lambda Y$ & $1,+\Gamma 1$ & $\cdot, \Lambda \Gamma T$ & $9+$ \\
\hline $1,1,10$ & $1,+490$ & $\cdot, \lambda \Gamma 10$ & 90. \\
\hline $1,+11$, & $1,+\mathrm{Y}$. & , , Аा & $1, \ldots$ \\
\hline
\end{tabular}

المصدر: جمعت من عينة الدراسة الميدانية وحسبت من نتائج تحليل هيكل تكاليف تحلية المياه

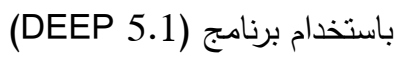


ع-تقدير تأثير السعة الإنتاجية لمحطة التحلية على تكلفة وحدة المياه المحلاة: تتأثر تكلفة وحدة المياه المنتجة بحجم السعة الانتاجية لمحطة التحلية، ووفقاً للمنطق الاقتصادي فإنها لإنه الإنها كلما زاد حجم الإنتاج انخفضت تكلفة إنتاج الوحدة، ويوضح الجدول رقم (0) أثز اختلاف التهاه السعة الانتاجية لمحطة التحلية على تكلفة انتاج وحدة المياه المحلاة، مع افتراض ثنات الاض التهات

$$
\text { باقي العوامل المؤثرة على التكلفة. }
$$

جدول رقم(؟): أثز اختلاف السعة الإنتاجية لمحطات التحلية على تكلفة وحدة المباه المحلاة

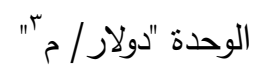

\begin{tabular}{|c|c|c|c|}
\hline \multicolumn{3}{|c|}{ 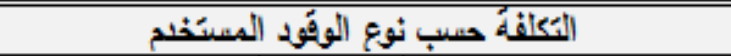 } & \multirow{2}{*}{ 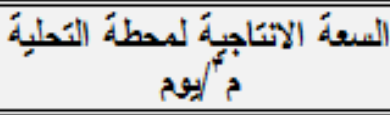 } \\
\hline فوتَو فوإطبِة (PV) & عنولزر. & وقُوند نوقى (Nuclear) & \\
\hline 1,001 & $1,0,$. & $1, r+r$ & 1. \\
\hline $1, \varepsilon 7 T$ & $1, E 1 Y$ & $1, Y 1 \varepsilon$ & r. \\
\hline $1,\{Y \mid$ & $1, r v \cdot$ & $1,1 V Y$ & $\Gamma \cdot$ \\
\hline $1, Y 97$ & $1, Y \leqslant 0$ & $1,+\varepsilon V$ & $\varepsilon$. \\
\hline $1, r T_{1}$ & $1,1 \%$. & $\cdot, 9 \vee r$ & 0. \\
\hline $1, Y 11$ & 1.17. & $0,97 Y$ & 7. \\
\hline 1,171 & $1,11 \mathrm{~V}$ & 0,919 & $\mathrm{r}$. \\
\hline 1,157 & 1,010 & $\cdot, M \Lambda V$ & ᄉ. \\
\hline 1,111 & $1,+7$ & $\cdot, \Lambda T Y$ & 9 . \\
\hline 1,91 & $1,+\varepsilon$. & $\cdot, \wedge \varepsilon Y$ & $1,$. \\
\hline $1,+11$ & $1,+5$. & $\cdot, \lambda \Gamma r$ & 11. \\
\hline 1,71 & $1, .10$ & $\cdot, \lambda 1 \mathrm{~V}$ & Ir. \\
\hline 1,01 & $1, \ldots r$ & $\cdot, \lambda \cdot \varepsilon$ & $1 \pi$. \\
\hline $1, E Y$ &., 991 &.,$V 9 T$ & $1 \varepsilon$. \\
\hline $1,+r T$ & $\cdot, 9 \lambda Y$ & $\cdot, \vee \wedge \varepsilon$ & 10. \\
\hline
\end{tabular}

المصدر: جمعت من عينة الدراسة الميدانية وحسبت من نتائج تحليل هيكل التكاليف باستخدام برنامج

(DEEP 5.1)

يتضح من نتائج الجدول السابق أن زيادة السعة الانتاجية لمحطات لتحلية من • امَّ/يوم

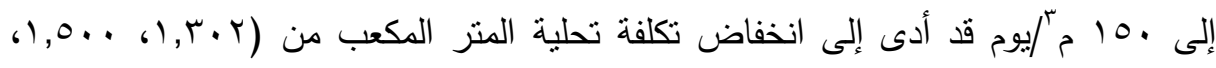




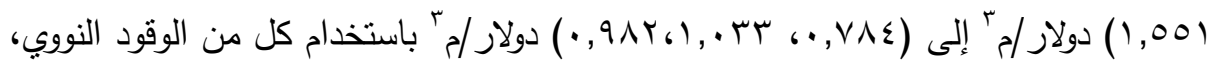

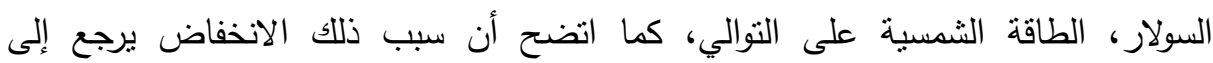
انخفاض نصيب المتز المكعب المنتج من التكلفة الرأسمالية لمحطة التحلية. ه - تقدير العلاقة الرياضية بين تكلفة انتاج وحدة المياه المحلاة والعوامل المؤثرة فيها: يعتبر اختيار النموذج الرياضي المعبر عن العلاقة بين التكاليف الانتاجية ومجموعة لهين العوامل المؤثرة فيها من المشاكل التي تعترض الباحثين عند اجراء الدراسات الاقتصادية القياسية نظراً لتعدد النماذج الرياضية التي يمكن استخدامها لتمثيل تلك العلاقة، وبالنظر إلى إنى

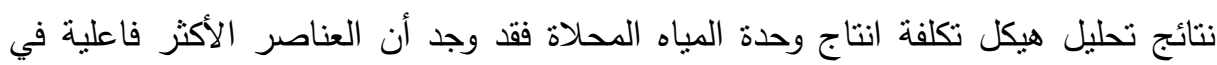
التأثير عليها هي الأربعة عناصر السابق ذكرها، وبناء على النتائج السابقة اتضح أن الأسلوب الاحصائي الأمثل لتمثيل تلك العلاقة، هو الانحدار الخطي البسيط Simple Linear) Regression Analysis)

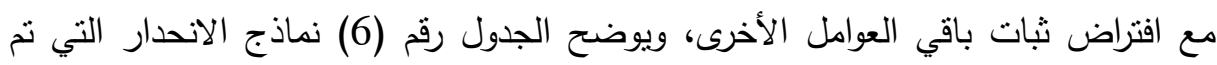
تقديرها لتفسير العلاقة بين المتغير التابع (Y) (التكلفة) والمتغيرات المستقلة , X (درجة ملوحة مياه المصدر، درجة حرارة مياه المصدر، نسبة نركيز الأملاح في المياه

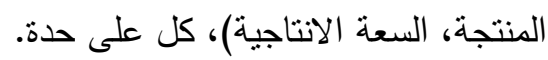


مجلة العلوم البيئية

معهد الدراسات والبحوث البيئية - جامعة عين شمس له

جدول رقم (•): معادلات نماذج الانحدار الخطي البسيط

\begin{tabular}{|c|c|c|c|c|c|c|}
\hline Sig. & $\mathbf{R}^{2}$ & $F$ & المعادلة & م & الوقوقودِ & المعاملكت \\
\hline 0.000 & 0.969 & $=681.276$ & $\begin{array}{l}Y=0.656+0.006 \mathrm{X}_{1} \\
\Leftrightarrow(114.962) \quad \approx(26.101)\end{array}$ & 1 & نوتى & \multirow{3}{*}{ مباه المصدة ملوحة } \\
\hline 0.000 & 0.979 & $\because 1023.710$ & $\begin{aligned} & Y=0.778+0.008 X_{1} \\
\Leftrightarrow & (114.566) \quad \Leftrightarrow(31.995)\end{aligned}$ & 2 & سولاز & \\
\hline 0.000 & 0.980 & $\because 1080.287$ & $\begin{aligned} & Y=0.806+0.008 X_{1} \\
\Leftrightarrow & (112.555) \quad \Leftrightarrow(32.868)\end{aligned}$ & 3 & ط طُمبةّة & \\
\hline 0.000 & 0.990 & $=2074.38$ & $\begin{aligned} & Y=0.884-0.002 X_{2} \\
\Leftrightarrow & (688.778) \Leftrightarrow(-45.545)\end{aligned}$ & 4 & نووى & \multirow{3}{*}{ مباه المصرة حرارة } \\
\hline 0.000 & 0.975 & $\because 806.784$ & $\begin{array}{c}Y=1.108-0.004 \mathrm{X}_{2} \\
\because(323.984) \quad \approx(-28.404)\end{array}$ & 5 & سولاز & \\
\hline 0.000 & 0.960 & $\because 502.619$ & $\begin{aligned} & Y=1.161-0.004 \mathrm{X}_{2} \\
\approx & (265.26) \Leftrightarrow(-22.419)\end{aligned}$ & 6 & طُمبقة & \\
\hline 0.000 & 0.995 & $=3336.511$ & $\begin{aligned} & Y=0.845-0.001 X_{3} \\
\approx & (5864.81) \quad \because(-57.763)\end{aligned}$ & 7 & نوتوى & \multirow{3}{*}{ نألمبة تركيز } \\
\hline 0.000 & 0.995 & $=3336.511$ & $\begin{array}{c}Y=1.043-0.001 X_{3} \\
\because(7239.059) \quad \because(-57.763)\end{array}$ & 8 & سولار & \\
\hline 0.000 & 0.993 & $=2596.435$ & $\begin{array}{c}Y=1.094-0.001 X_{3} \\
\ldots(7418.913) \approx(-50.955)\end{array}$ & 9 & طُطمبنة & \\
\hline 0.000 & 0.866 & $\because 84.165$ & $\begin{array}{c}Y=1.222-0.003 \mathrm{X}_{4} \\
\Leftrightarrow(41.676) \quad \Leftrightarrow(-9.174)\end{array}$ & 10 & نوتى & \multirow{3}{*}{ 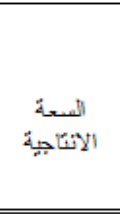 } \\
\hline 0.000 & 0.866 & $=84.165$ & $\begin{aligned} & Y=1.420-0.003 X_{4} \\
\Leftrightarrow & (35.866) \quad \because(-9.174)\end{aligned}$ & 11 & سولاز & \\
\hline 0.000 & 0.866 & $=84.165$ & $\begin{array}{c}Y=1.471-0.003 X_{4} \\
\ldots(43.172) \quad \approx(-9.174)\end{array}$ & 12 & طُمَفِّة & \\
\hline
\end{tabular}

المصدر: من اعداد الطالب من خلال تحليل الاتحدار البسيط باستخدام برنامج SPSS من نتائج الجدول السابق يتضح لنا ما يلي:

1- نموذج الانحدار لعلاقة اختلاف درجة ملوحة مياه المصدر بتكلقة وحدة المياه المحلاة

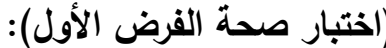

(أ) في حال استخدام الوقود النووي تبين ما بلي: الئي

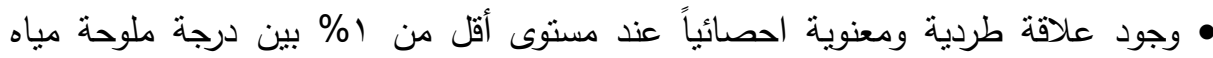

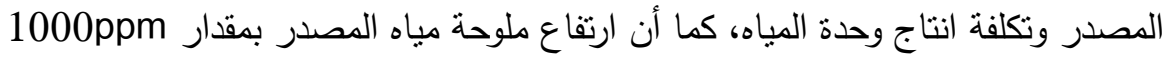

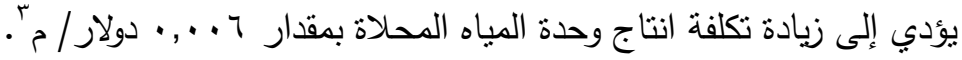


• قيمة معامل التحديد (R2) تساوي 97,9\%، بما يعني أن المتغير المستقل (درجة ملوحة مياه المصدر) يفسر 9,99\% من التغيرات التي تحدث في المتغير التابع (تكلفة وحدة

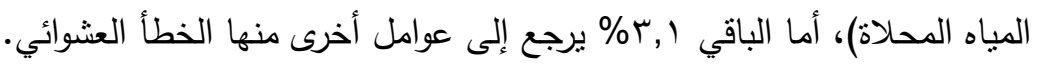

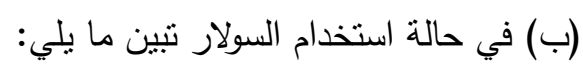

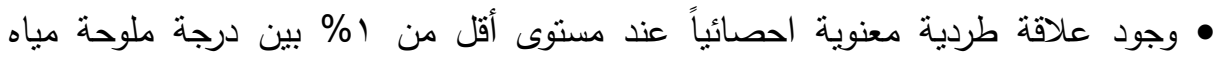
الدصدر وتكلفة انتاج المتر المكعب من المياه المحلاة، كما أن ارتفاع ملوحة مياه المصدر بمقدار 1000ppm يؤدي إلى زيادة تكلفة انتاج وحدة المياه المحلاة بمقدار

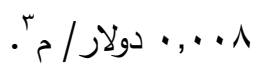

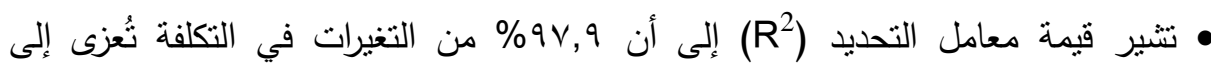
اختلاف درجة ملوحة مياه المصدر .

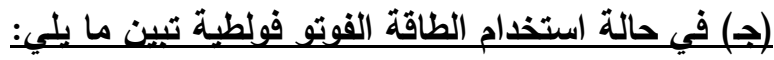

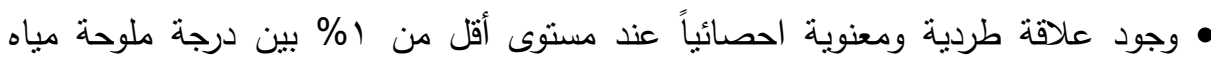
المصدر وتكلفة انتاج المتر المكعب من المياه المحلاة، كما أن ارتفاع ملوحة مياه المصدر بمقدار 1000ppm يؤدي إلى زيادة تكلفة انتاج وحدة المياه المحلاة بمقدار

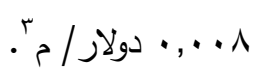

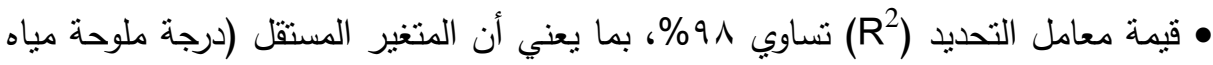
المصدر) يفسر 9 9,9\% من التغيرات التي تحدث في المتغير التابع (تكلفة وحدة المياه

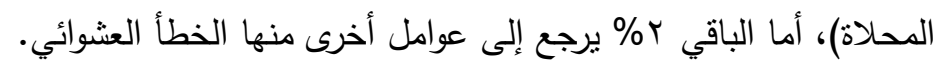

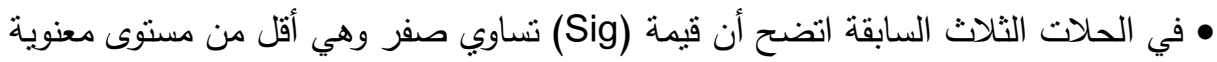

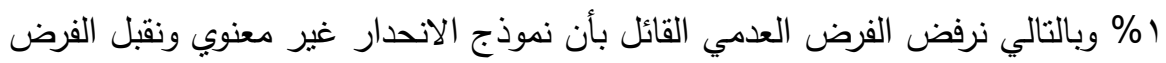

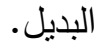


نموذج الانحار المقدر لعلاقة اختلاف درجة حرارة مياه المصدر بتكلقة وحدة المياه المحلاة (اختبار صحة الفرض الثاني): (أ) في حال استخدام الوقود النووي تشبير بيانات المعادلة رقم (ع) إلى ما بلي:

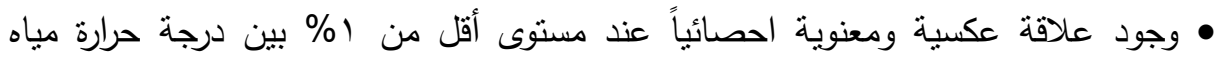
المصدر وتكلفة انتاج المتر المكعب من المياه المحلاة، كما أن ارتفاع درجة مياه المصديه اهدر

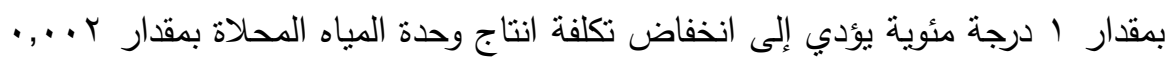

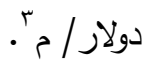

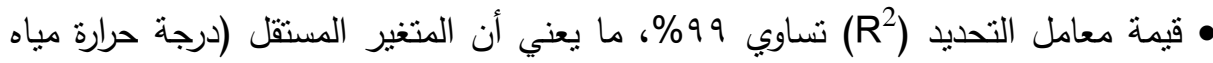
المصدر) يفسر 99\% من التغيرات التي تحدث في المتغير التابع (تكلفة وحدة المياه المحلاة)، أما الباقي ( \% يرجع إلى عوامل أخرى منها الخطأ العشوائي.

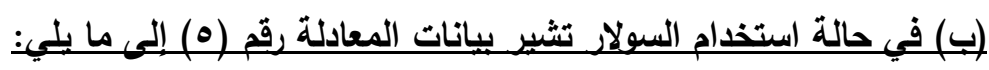

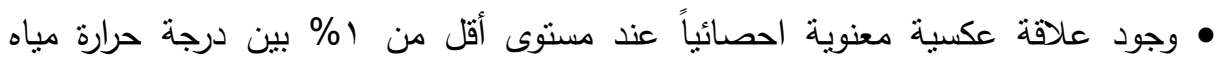
المصدر وتكلفة انتاج وحدة المياه المحلاة، كما أن ارتفاع درجة حرارة مياه المصدر بمقدار دئيار | درجة مئوية يؤدي إلى انخفاض تكلفة انتاج وحدة المياه المحلاة بمقدار ع ـ .., ـ دولار /

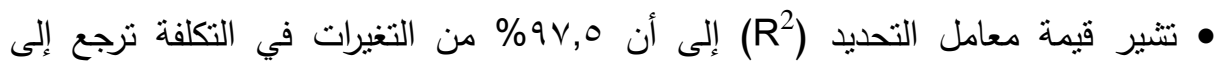
اختلاف درجة حرارة مياه المصدر والباقي يعزى إلى عوامل أخرى منها الخطأ العشوائي.

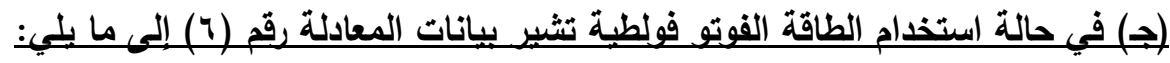
• وجود علاقة عكسية معنوية عند مستوى أقل من ا \% بين درجة حرارة مياه المصدر وتكلفة انتاج المتر المكعب من المياه المحلاة، كما أن زيادة درجة حرارة مياه المصدر بمقدار درجة

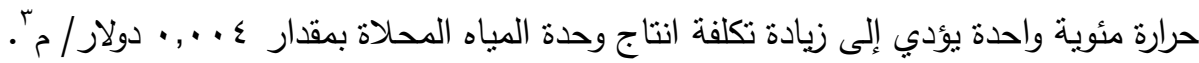

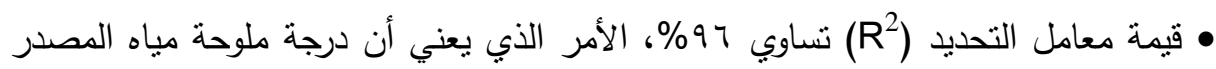

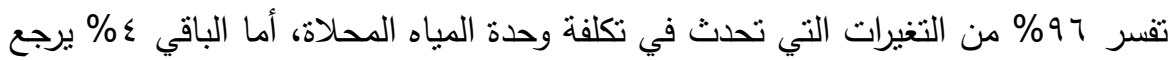
إلى عوامل أخرى منها الخطأ العشوائي. 
• في الحلات الثلاث السابقة اتضح أن قيمة (Sig) تساوي صفر وهي أقل من مستوى معنوية

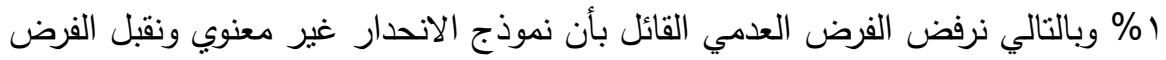
البديل.

r-نموذج الانحدار لعلاقة نسبة تركيز الأملاح في المياه المنتجة بتكلفة وحدة المياه المحلاة (اختبار صحة الفرض الثالث):

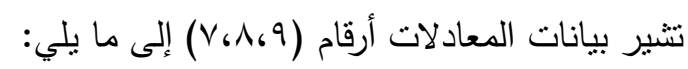
• وجود علاقة عكسية معنوية عند مسنوى أقل من ا ٪ بين ارتفاع تركيز الأملاح في المياه المنتجة وتكلفة انتاج المتر المكعب من المياه المحلاة، كما أن ارتفاع نسبة نركيز الأملاح 50 ppm وذللك بالنسبة للثناثة أنواع المختلفة من الوقود.

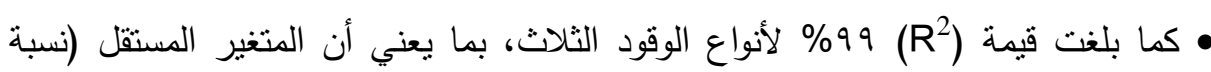
تركيز الأملاح في المنتجة) يفسر 99\% من التغيرات التي تحدث في المتغير التابع (تكلفة وحدة المياه المحلاة)، كما ثنتت معنوية النماذج عند مستوى أقل ( الفرض العدمي القائل بأن نموذج الانحدار غير معنوي ونقبل الفرض البداه البديل. r-نموذج الانحدار للعلاقة بين زيادة السعة الانتاجية للمحطة وتكلفة وحدة المياه المحلاة (اختبار صحة الفرض الرابع):

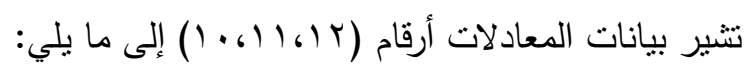

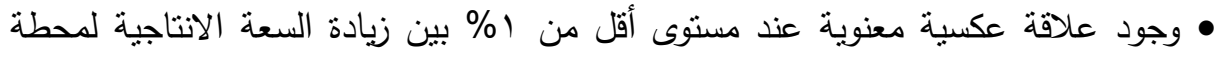

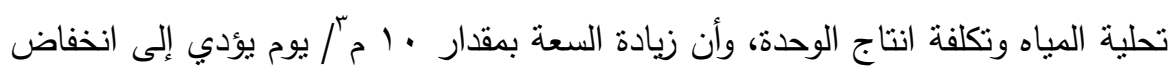

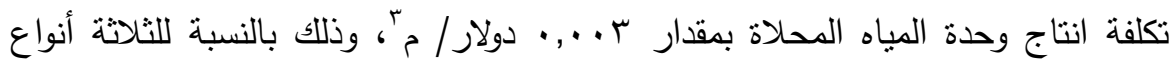

$$
\text { المختلفة من الوقود. }
$$

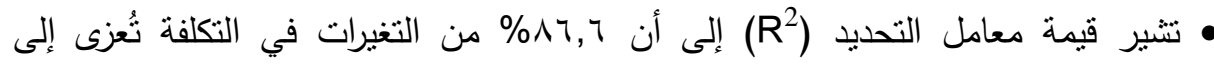

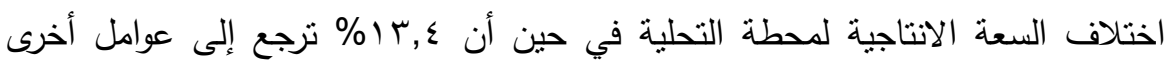

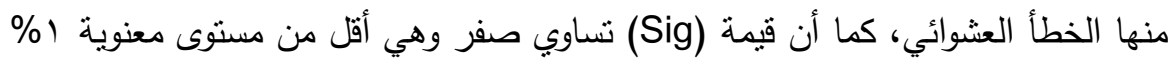


وبالتالي نرفض الفرض العدمي القائل بأن نموذج الانحدار غير معنوي ونقبل الفرض البديل.

ومن هنا نخلص إلى أنه لا يمكن تحديد سعر ثابت لتكلفة انتاج وحدة المياه المحلاة

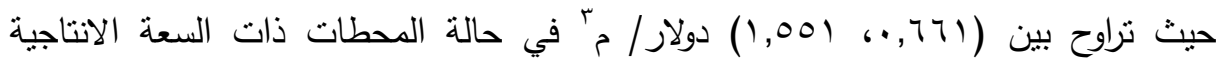
الصغيرة المخصصة لتحلية مياه الآبار عالية الملوحة (Brackish Water)، إذ إنان مأن الأمر

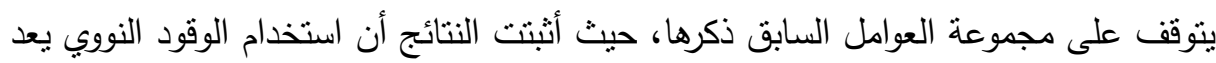
خياراً استراتيجياً وفعالاً نظراً لانخفاض تكلفته كما يمتاز بانخفاض آثاره السلبية على البيئة

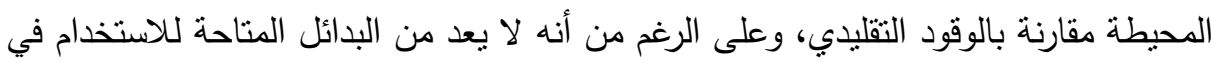

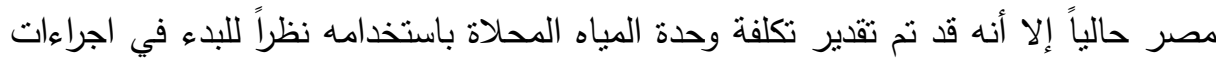

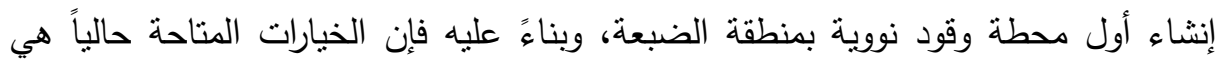

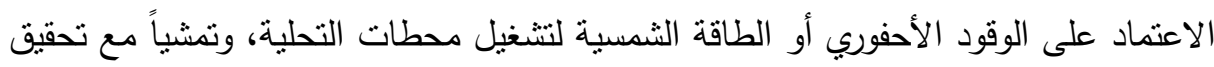

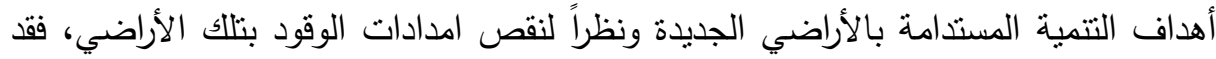
يكون الاعتماد على المصادر الطبيعية المتوفرة بتلك الأراضي كمصدر للطاقة اللازمة لتشغيل محطات التحلية هو الحل الأمثل لتوفير المياه العذبة للمجتمع الصحراوي وبتكلفة مناسبة. ثانياً: تقدير الربحية المالية لمشروع الإدارة المتكاملة لمحطة تحلية مصغرة تعمل بالطاقة لإنة المتجددة في منطقة الثلاتين (اختبار صحة الفرض الخامس): في محاولة تحقيق الاستفادة القصوى من مشروعات تحلية المياه بمنطقة الثلاتين والتي تستهدف في المقام الأول تحقيق العدالة الاجتماعية بتوفير مياه نقية صالحة للثرب لمجتمع لاهن الدراسة، فإن العمل على رفع الكفاءة الاقتصادية تحت مظلة الاستدامة الإيكولوجية يعد هدفاً آخر لإدارة ذلك المشروع بصورة متكاملة، حيث ينم تلافي الآثار البيئية السالبة لحقن المحلول

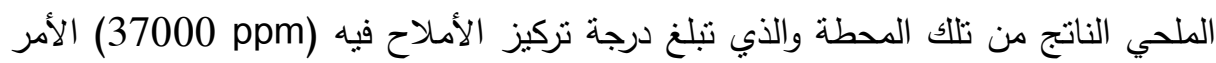
الذي قد يزيد من نملح الأراضي بالمنطقة، وإعادة استخدامه بعد اجراء عمليات المعالجة اللازمة لاستزراع الجمبري بالاعنماد على تقنية البيوفلوك وبالتالي رفع الكفاءة الاقتصادية

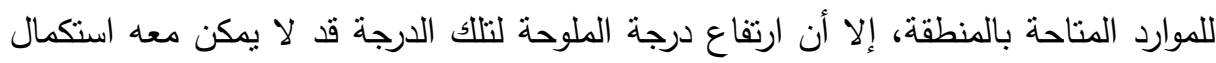


باقي النموذج المقترح للإدارة المتكاملة لمحطات تحلية المياه باستزراع المحاصيل العلفية ذلك الكي لتخطي ذللك المحلول للحدود القصوى لتحمل تلك النباتات لنسب الأملاح المركزة في المياه التي يمكن ريها بها، لذا فإنه يكتفي هنا بمرحلة الاستزراع السمكي، ولتقييم ربحية الاستثمار في هذا المجال يتم استخدام المعايير التالية: الفروض المستخدمة في التحليل المالي للمشروع: اعتمد التحليل على عدة ثوابت فرضية يقوم عليها تحليل الربحية التجارية للمشروع وهي كالتالي: ا-قدرت اجمالي التكاليف الرأسمالية وتكاليف التشغيل السنوية والايرادات الكلية لمشروع التشيه الإدارة المتكاملة لمحطة تحلية مياه مصغرة تعمل بالطاقة المتجددة وفقاً لمتوسط أسعار

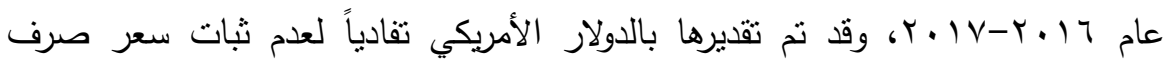

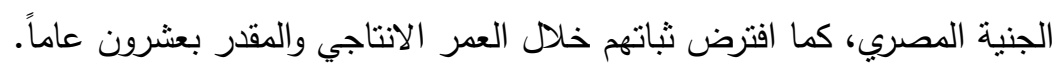

r-استخدم سعر خصم 10\% (وهو يمثل نفقة أفضل فرصة بديلة متاحة لاستثمار رأس المال

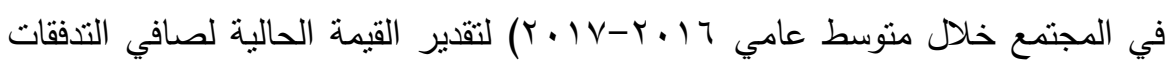
النقدية.

r-نم حساب القيمة التخريدية لوحدتي التحلية والطاقة وكذلك الآلات والمعدات على أساس أنها تساوي • (\% من قيمتها الحالية، تضاف قيمتها ضمن التدفقات النقدية للعام العشرون.

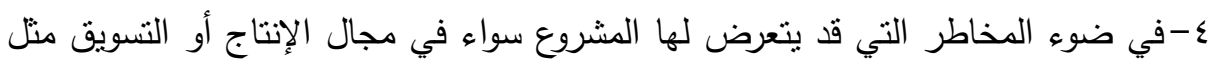
انخفاض الإنتاجية وارتفاع أسعار مسنلزمات الإنتاج أو انخفاض أسعار المنتج، لذاء

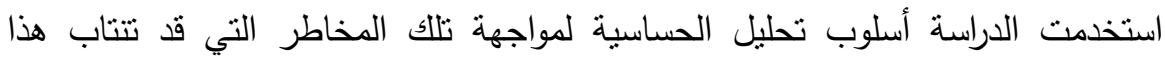
النشاط، حيث افترضت الدراسة حدوث زيادة بواقع · (\% للتكاليف التي بني عليها التحليل، أو انخفاض العائد بنفس النسبة، وأخيراً احتمال حدوث الاثنين معاً. 
وتوضح بيانات الجدول رقم (T) تقديرات معايير التحليل المالي للمشروع كالتالي:

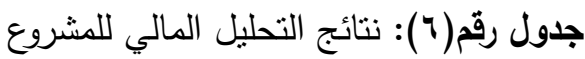

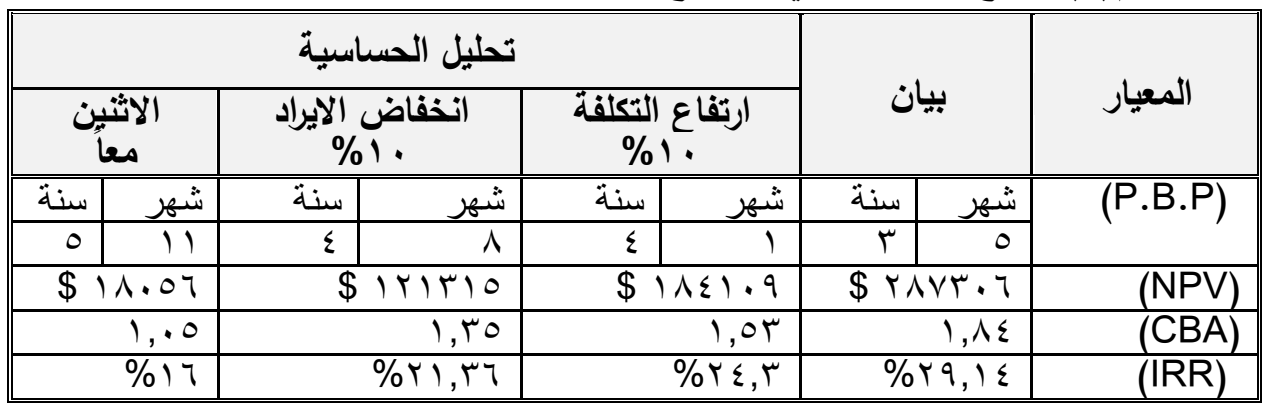

المصدر: نتائج تحليل بيانات الدراسة الميدانية باستخدام برنامج Cost Benefit Analysis.

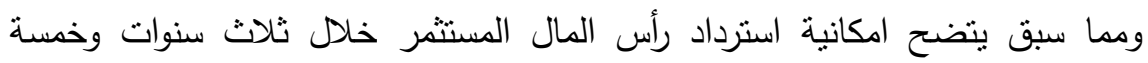

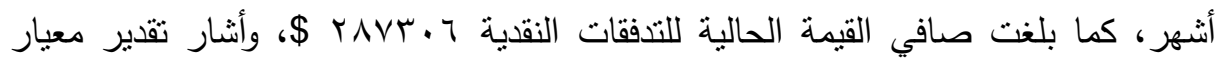

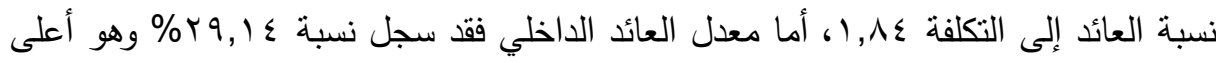
من تكلفة الفرصة البديلة المتاحة في مجتمع استثمار رأس المال والتي بمثلها متوسط سعر

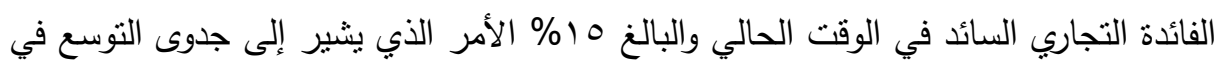
اقامة تلك الأنشطة، في حين تشير تقديرات معايير التقييم المالي وفقاً لتحليل الحساسية (زيادة

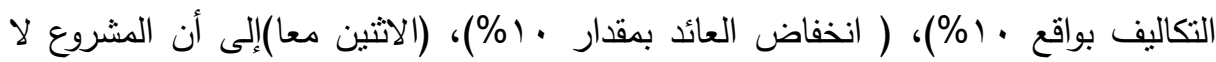

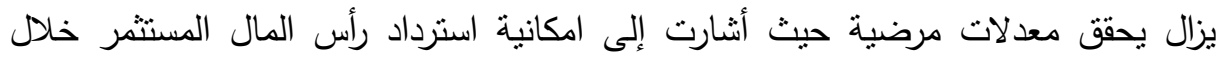
فترات (أربع سنوات وشهر، أربع سنوات وثمانية أشهر، خمس سنوات اتفات واحدى عشر شهراً)،

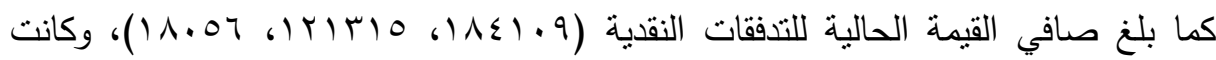

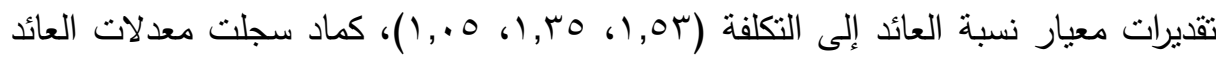

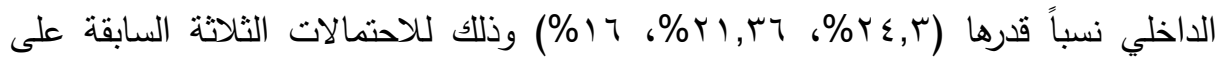
التوالي، ومما سبق أنه بقياس أثر التغيرات المحتملة في المتغيرات الاقتصادية على كفاءة الاستثمار في ذلك النشاط فإنه لا يزال يحقق معدلات مرضية بالنسبة لمؤشرات التقييم المستخدمة الأمر الذي يؤكد على سلامة الاستثمار في الوحدات الانتاجية محل الدراسة. 
استهدف البحث دراسة اقتصاديات إدارة المحطات المصغرة لتحلية مياه الآبار عالية الملوحة بصورة متكاملة تحقيقاً لأغراض التتمية المستدامة بالأراضي الصحراوية، وذلك من خلال بناء نماذج محاكاة (Simulation Models) لتقدير تكلفة وحدة المياه المحلاة بمعلومية مجموعة المتغيرات المستقلة المؤثرة فيها (درجة ملوحة مياه المصدر ، درجة ملوحة

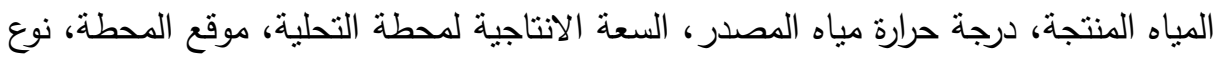

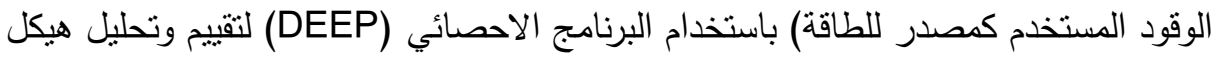

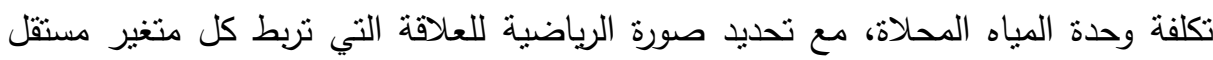

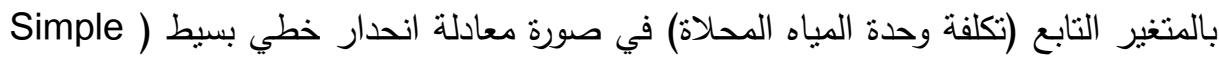
(Linear Regression Equation انتاج وحدة المياه المحلاة، اخيراً تم استخدام أسلوب التحليل المالي لتقدير الربحية التجارية لنموذج مشروع الإدارة المتكاملة لمحطة تحلية مياه تعمل بالطاقة المتجددة بمنطقة الثلاتين

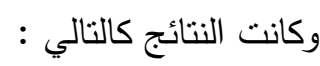
1-بالنسبة للتحليل الاقتصادي لتكلفة وحدة المباه المحلاة تبين أن هنالك مجموعة من العوامل أثرت على تكلفة انتاج وحدة المياه المحلاة على النحو التالي:

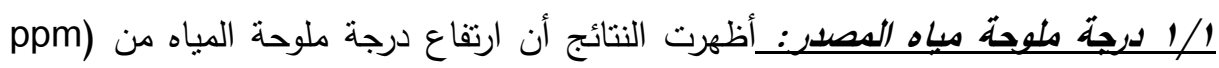

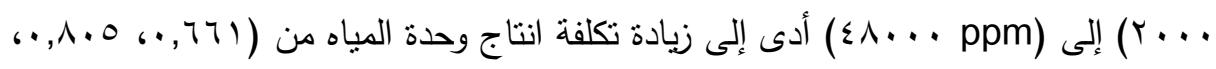

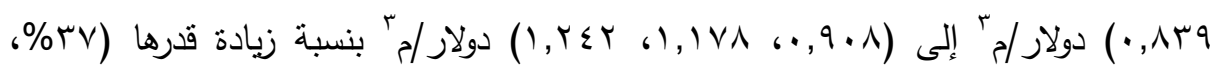

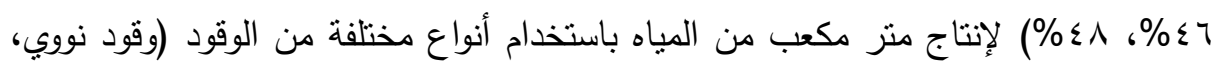

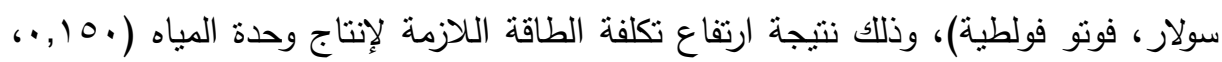

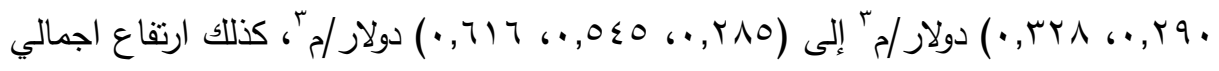

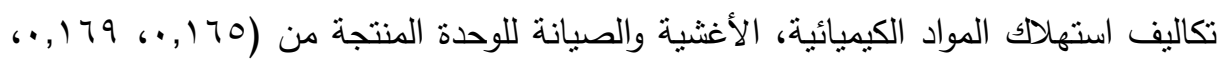

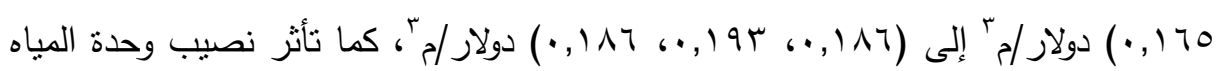

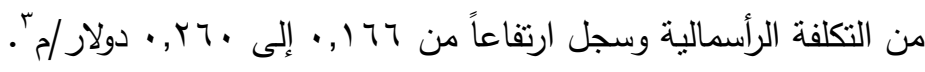


وبتقدير نماذج الانحدار لتلك العلاقة تنين وجود علاقة طردية ومعنوية احصائياً عند

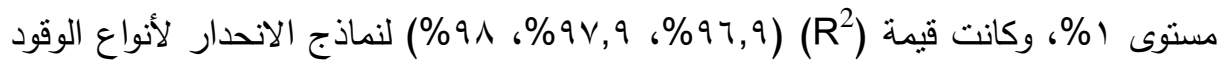
(النووي، السولار ، الفوتو فولطية) على النوالي.

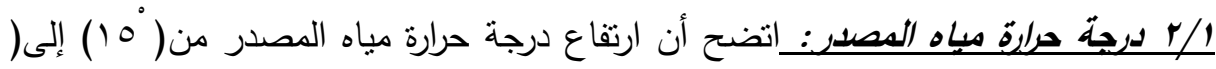

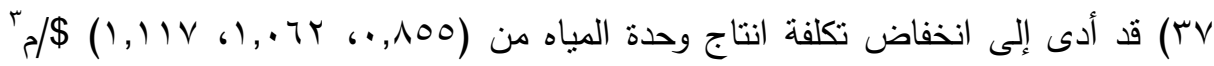

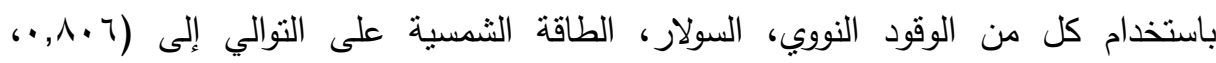

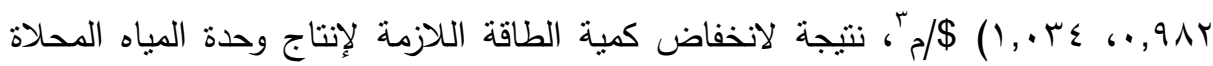

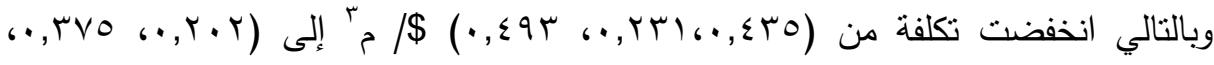
. دولار/مّ، وعند تقدير نماذج الانحدار لتلك العلاقة تبين وجود علاقة عكسية ومعنوية احصائياً

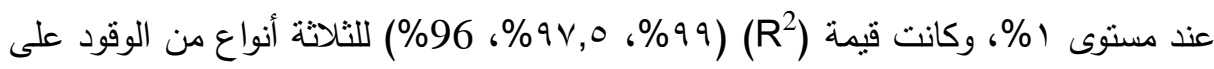
التوالي. 1\%/ نسب تركيز الأملاح في المياه المنتجة: اتضح أن زيادة درجة نركيز الأملاح في المياه

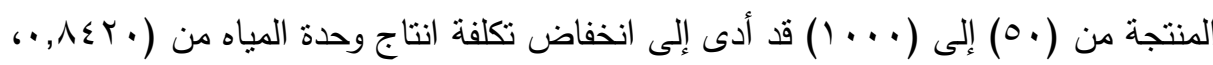

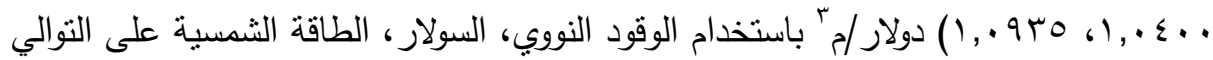

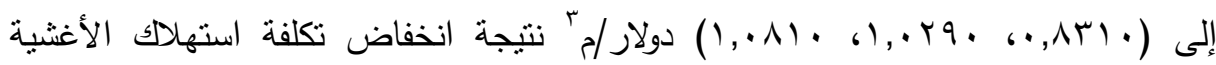
المستخدمة في حال انتاج مياه بتركيز أملاح أعلى، بالإضافة إلى انخفاض في كمية الطاقة

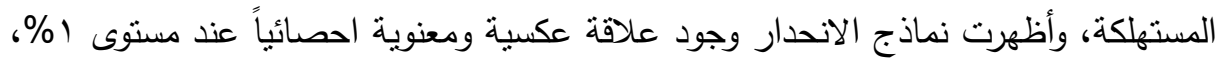
وسجلت قيم (R²\%) (99\%) لنماذج الاتحدار لأنواع الوقود الثناث.

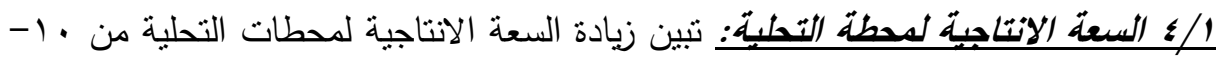

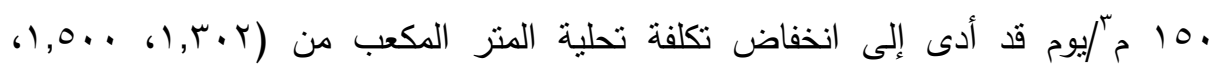

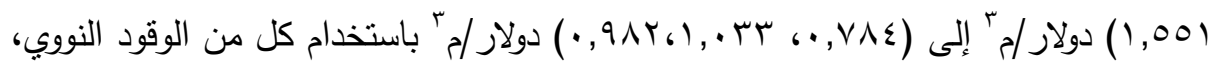
السولار، الطاقة الثمسية على التوالي، نتيجة لانخفاض نصيب وحدة المياه من التكلفة الرأسمالية لمحطة التحلية، وقد أكدت نماذج الانحدار على وجود علاقة عكسية معنوية 


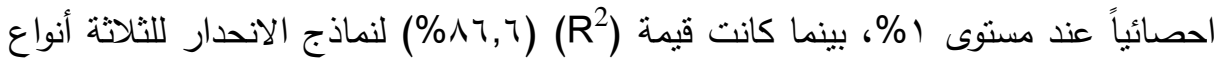
من الوقود.

r-تقدير الريحية المالية لمشروع الإدارة المتكاملة لمحطة تحلية مصفرة تعمل بالطاقة

\section{المتجددة في منطقة الشلاتين}

أظهرت نتائج التحليل المالي أن فترة استرداد رأس المال كانت ثلاث سنوات وخمسة

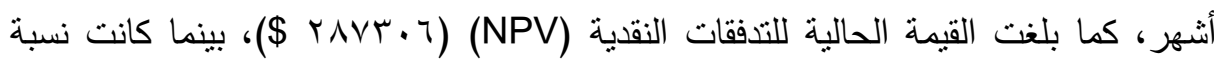
المنفعة إلى التكلفة (CBA) ( (C乏, ())، أما معدل العائد الداخلي (IRR) فكانت نسبته

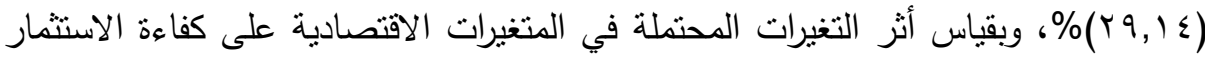

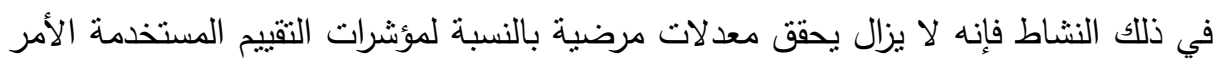
الذي يؤكد على سلامة الاستثمار في الوحدات الانتاجية محل الدراسة.

\section{التيوكيايت}

في ضوء ما توصل إليه البحث من نتائج بشكل عام، وبخصوص تتمية العديد من

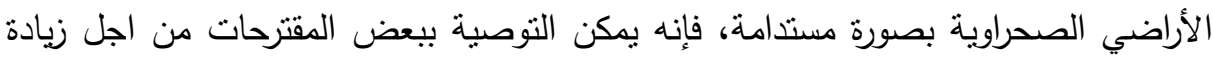

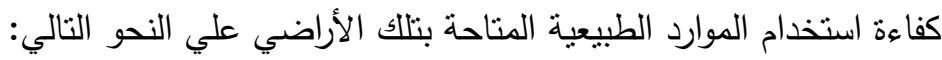
1-إدارة المشروعات التتموية بصفة عامة ومشروعات الموارد المائية بصفة خاصة بصورة متكاملة يراعى فيها رفع الكفاءة الاقتصادية وتحقيق العدالة الاجتماعية تحت مظلة

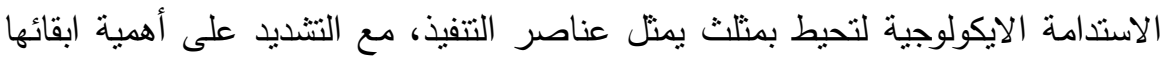

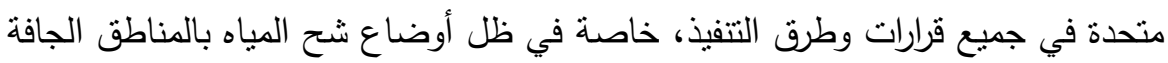
وشبة الجافة.

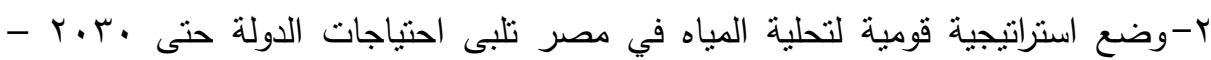

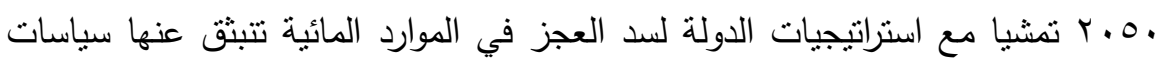
تتفيذية وأطر مؤسسية وتمويلية ويتم على أساسها وضع الخطط والمشروعات. 
r-تكاتف جهود كل الجهات والهيئات المعنية بتحلية المياه في مصر لوضع خريطة تحلية المياه بمصر وتحديد مناطق مآخذ المياه المسنوفاة للشروط البيئية والصحية، مع التحديد الاقيق للخصائص الطبيعية لتلك المياه. ع - التوسع في استغلال الطاقات الجديدة والمتجددة في تشغيل محطات التحلية. 0- عمل دراسات لآليات تطوير قرارات التصنيع المحلي لمكونات محطات التحلية. 1-توسيع الاستفادة من ناتج محطات التحلية من المياه عالية الملوحة لزيادة إنتاجية المحطة لهُبة وتعظيم العائد الاقتصادي، مع حماية البيئة من التلوث الناتج من صرف المن المياه المركزة، وذلك بإنشاء مجموعة من المشروعات ذات الصلة الأمامية والخلفية بصناعة التحلية.

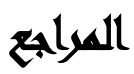

أويس عطوه الزنط(ب991): مناهج استخدام الثقنية الحديثة في التحليل الاقتصادي لأسس تقييم المشروعات ودراسات الجدوى، المكتبة الأكاديمية

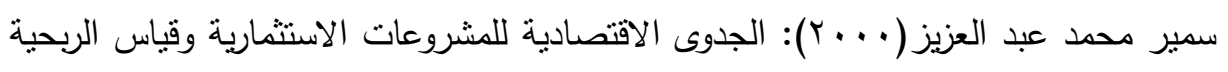
التجارية والقومية،مكتبة الإشعاع، الإسكندرية

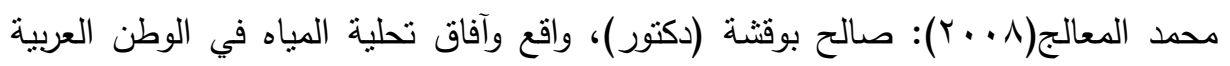

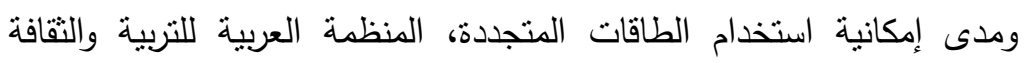

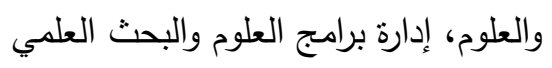

محمد منير مجاهد(Y ( ب): وآخرون، مصادر المياه في مصر وآفاق تتميتها، المكتبة الأكاديمية القومية

أحمد محمد على يوسف(Y V Y r): وآخرون خارطة طريق مستقبل التحلية في مصر، نقرير

$$
\text { غير منشور ، مركز بحوث الصحراء }
$$

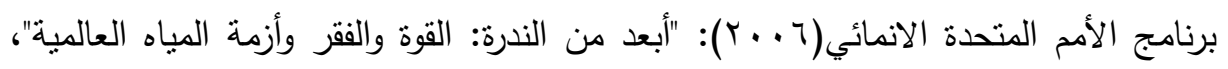

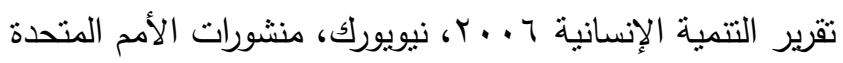


عبير فرحات علي وآخرون

$$
\begin{aligned}
& \text { كريستوف برانيه، دانيال فالنسويلا، الإدارة المتكاملة للموارد المائية في أحواض الأنهار }
\end{aligned}
$$

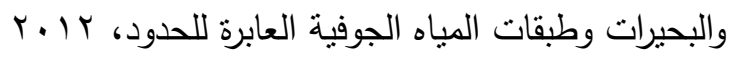

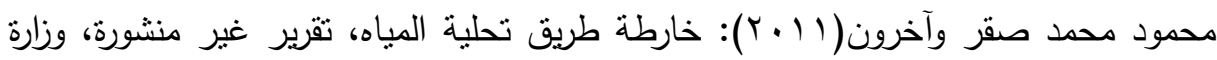

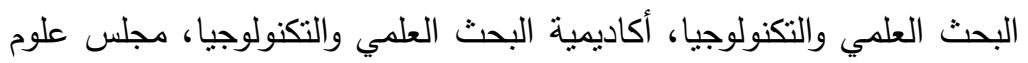

$$
\begin{aligned}
& \text { المياه }
\end{aligned}
$$

Agarwal et al. Integrated Water Resource Management, Global water partnerships, Technical Advisory Committee, 2000.

Asmerom M. Gilau \& Mitchell J. Small, Designing Cost Effective Sea Water Reverse Osmosis System Under Optimal Energy Options for Developing Countries, Carnegie Mellon University, Civil \& Environmental Engineering and public Policy, Proceeding of the International Conference of Renewable Energy of Developing Countries, 2006.

B. S. Richards and A. I. Schafer, Renewable Energy Powered Water Treatment Systems, Sustainability Science and Engineering, vol. 2, no. Sustainable Water for the Future: Water Recycling Versus Desalination, 2010.

C. Li, Y. Goswami and E. Stefanakos, Solar assisted sea water desalination: Review, Renewable and Sustainable Energy Reviews, vol. 19, pp. 136-163, December 2013.

Dalia E. Abo Zaid, Economic analysis of a stand-alone reverse osmosis desalination unit powered by photovoltaic for possible application in the northwest coast of Egypt, Desalination and Water Treatment, DOI, 10.1080/19443994.2014.911704,April 2014.

Hisham T. El-Dessouky, Hisham M. Ettouney, Fundamentals of Salt water Desalination.

Hafez, S. El-Manharawy, Economics of seawater RO desalination in the Red Sea region, Egypt, Part 1.A case study, Desalination 153, pp. 335-347, (2002).

International Atomic Energy Agency, Examining the economics of seawater desalination using the DEEP Code, Nuclear power Technology Development Section, Vienna, Austria, November, 2000.

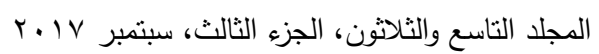


Mirei Isaka \& Giorgio Simbolotti, Water Desalination Using Renewable Energy, IEA, ETSAP and IRENA, Technology Policy Brief I 12 January, 2013.

M. Shatat, M. Worall and S. Riffat, Opportunities for Solar Water Desalination World Wide Review, Sustainable Cities and Society, Vol.9, 2013.

\title{
INTEGRATED MANAGEMENT ECONOMIES OF HIGH SALINITY WATER WELLS DESALINATION IN DESERT LANDS.
}

\author{
Aly, Abeer, F. ${ }^{(1)}$; EL.kassas, H. I. $^{(2)}$; Abo Zaid, Dalia. E. ${ }^{(3)}$ \\ and Abd Alaziz, M. O. ${ }^{(3)}$
}

1) Faculty of Commerce, Ain Shams Unevirsty. 2) Institute of Environmental Studies and Research , Ain Shams University 3) Desert Research Center.

\begin{abstract}
The research aims to reach for integrated management economies of small water desalination plants, to achieve sustainable development in the desert lands, by studying how to apply integrated management methods of high-salinity wells desalination plants, also estimate the cost of desalinated water unit produced from reverse osmosis desalination plants powered by solar energy by changing of independent variables that effect on cost finally, a financial analysis of the model of the integrated management project of a desalination plant powered by renewable energy in Al Shalatin area has been done, The main results were as follows:

(1): Increasing the salinity of water from (ppm 2000) to (ppm 48000) led to an increase in the cost of water unit production from $\$ 0.839$ $\$ 1.242 / \mathrm{m}^{3}$.

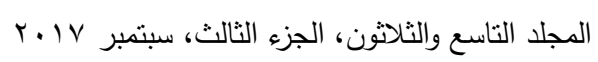


عبير فرحات علي وآخرون

(2): Increasing water temperature from (15) to (37) reduced the cost from $\$ 1.117-1.034 / \mathrm{m} 3$.

(3): Increased salinity concentration in water produced from 50 to 1000 ppm resulted in a lower cost of $1.0935-1.0810 \$ / \mathrm{m} 3$.

(4): Increasing the production capacity of the desalination plant from 10 $\mathrm{m} 3$ / day to $150 \mathrm{~m} 3$ / day led to a decrease in desalination cost of cubic meter of desalinated water from 1.551 to 1.033 \$.

(5): The financial profitability of the project shows that the payback period was (four years and five months), (NPV) was (46657.21 \$), the proportion of (CBA) (1.41), and (IRR) was $22.45 \%$, which indicates the profitability of investment in such projects.

Keywords: economics of desalination - reverse osmosis - water scarcity - simulation models. 